\title{
Algebraic conformal quantum field theory in perspective
}

\author{
KARL-HENNING REHREN \\ Institut für Theoretische Physik, Universität Göttingen, \\ Friedrich-Hund-Platz 1, D-37077 Göttingen, Germany \\ rehren@theorie.physik. uni-goettingen.de
}

\begin{abstract}
Conformal quantum field theory is reviewed in the perspective of Axiomatic, notably Algebraic QFT. This theory is particularly developped in two spacetime dimensions, where many rigorous constructions are possible, as well as some complete classifications. The structural insights, analytical methods and constructive tools are expected to be useful also for four-dimensional QFT.
\end{abstract}

\section{Contents}

1 Introduction

2 CFT in the context of relativistic QFT 2

2.1 Conformal symmetry ................. 3

2.2 Two dimensions . . . . . . . . . . . . . . . 5

2.3 Representations . . . . . . . . . . . . . 8

2.4 Different axiomatizations . . . . . . . . . . . . 9

3 Algebraic QFT 11

4 Algebraic CFT on the circle 13

4.1 Axioms . . . . . . . . . . . . . . . . . . . . 13

4.2 Superselection sectors . . . . . . . . . . . . . 16

5 Chiral model constructions

5.1 Free fields . . . . . . . . . . . . . . . . . 18

5.2 Wick products and Fermionization . . . . . . . . . . . 19

5.3 Bosonization ....................... 21 
5.4 Orbifold constructions ................... 21

5.5 Simple current extensions ................ 22

5.6 The coset construction ................. 22

6 Algebraic constructions (not only conformal) 23

6.1 Superselection sectors and symmetry . . . . . . . . . 23

6.2 Extensions by Q-systems ................ 2. 25

6.3 Borchers triples and deformation methods . . . . . . . 2 27

6.4 Holographic models . . . . . . . . . . . . . . . . 30

6.5 Phase boundaries .................. 32

7 Final Remarks 33

7.1 What is special about CFT in two dimensions? . . . . . . . 33

7.2 What can we learn for QFT in four dimensions? . . . . . . . 35

\section{Introduction}

We give an overview of the methods and results of conformal quantum field theory (CFT), accumulated in the last three decades, in the perspective of axiomatic approaches. In particular, we advocate the point of view that a CFT is just a relativistic quantum field theory (QFT) which is invariant under the group of conformal spacetime symmetries. Thus, there are no independent "CFT axioms", but the usual QFT axioms apply with an enlarged symmetry.

Starting from the Wightman axiomatic setting in Sect.2, we emphasize the crucial importance of inequivalent representations (superselection sectors) for several aspects of QFT. This motivates the formulation in the Haag-Kastler axiomatic setting ("algebraic quantum field theory", AQFT), which is particularly powerful to address superselection sectors. We give a brief review of this setting in Sect. 3, and turn to its specific application to chiral CFT in Sect.4,

The sections Sect. 5 and Sect. 6 give an overview of various constructive methods to produce models of CFT (and QFT), and related classification results.

\section{CFT in the context of relativistic QFT}

Since the conformal group contains the proper orthochronous Poincaré group, a conformal QFT is in particular a relativistic QFT in the usual sense. The sim- 
plest examples are the massless Klein-Gordon and Dirac fields and the Maxwell field in four spacetime dimensions (4D). Their conformal symmetry arises as a consequence of the massless field equations, and not because it was "postulated" as an extra feature.

The Wightman axioms describe quantum fields $\phi$ as operator-valued distributions on (a common invariant domain in) a Hilbert space $\mathcal{H}$, subject to the principle of Locality (Einstein Causality $=$ commutation at spacelike distance). The Hilbert space carries a unitary representation $U$ of the Poincaré group $P_{+}^{\uparrow}=\mathrm{SO}(1, D-1)_{0} \ltimes \mathbb{R}^{1, D-1}$ in $D$ dimensions, which extends to a representation of the conformal group $\operatorname{Conf}_{D}$ (see below). Conformally covariant fields transform with a transformation law

$$
U_{g} \phi(x) U_{g}^{*}=\alpha_{g}(\phi(x))=D(g, x)^{-1} \phi(g x)
$$

where the fields may be multiplets, and accordingly $D(g, x)$ is a suitable matrixvalued cocycle. Finally, the vacuum state is a unique vector $\Omega \in \mathcal{H}$ which is a zero-energy ground state for the Hamiltonian (the generator of the subgroup of time-translations) in every Lorentz frame, which implies that it is invariant under $U_{g}$ for all $g \in \operatorname{Conf}_{D}($ in $D>2$ ).

For Poincaré and scale transformations $g_{\lambda}: x \mapsto \lambda x\left(\lambda \in \mathbb{R}_{+}\right)$, the cocycle $D(g, x)$ is independent of $x$ and is just a matrix representation of $(\mathrm{SO}(1, D-$ $\left.1)_{0} \times \mathbb{R}_{+}\right) \ltimes \mathbb{R}^{1, D-1}$. For scale transformations, one has $D\left(g_{\lambda}\right)^{-1}=\lambda^{d}$ where the parameter $d \geq 0$ is the scaling dimension of the field $\phi$.

\subsection{Conformal symmetry}

We take the conformal group $\operatorname{Conf}_{D}$ to be the connected component of the group of transformations of $D$-dimensional Minkowski spacetime that preserve the Minkowski metric $d s^{2}=\eta_{\mu \nu} d x^{\mu} d x^{\nu}$ up to a positive factor $\omega^{2}(x)$ that may depend on $x$. This group is generated (in $D>2$ ) by the translations

$$
T_{a}: \quad x^{\mu} \mapsto x^{\mu}+a^{\mu} \quad\left(a \in \mathbb{R}^{1, D-1}\right)
$$

and the involutive "conformal inversion"

$$
I: \quad x^{\mu} \mapsto \frac{x_{\mu}}{(x \cdot x)}
$$

such that $S_{b}=I \circ T_{b} \circ I$ are the special conformal transformations

$$
S_{b}: \quad x^{\mu} \mapsto \frac{x^{\mu}-(x \cdot x) b^{\mu}}{1-2(x \cdot b)+(x \cdot x)(b \cdot b)} \quad\left(b \in \mathbb{R}^{1, D-1}\right) .
$$


Proper orthochronous Lorentz transformations $x \mapsto \Lambda x$ and scale transformations $x \mapsto \lambda x$, as well as the inversion $I$ are generated by translations and special conformal transformations.

The singularity of the conformal inversion and the special conformal transformations can be dealt with as follows (see, e.g., [56, 13]). One introduces the "Dirac manifold" which is the projective null cone $\left\{\xi \in \mathbb{R}^{2, D}: \xi \cdot \xi=\right.$ $0\} / \xi=\lambda \xi, \lambda \neq 0 \simeq\left(S^{1} \times S^{D-1}\right) / \mathbb{Z}_{2}$ in an auxiliary space with metric $(+,-\cdots-,+)$. $S^{1}$ is timelike, $S^{D-1}$ spacelike. The conformal group acts perfectly regular as Conf $_{D} \sim \mathrm{SO}(2, D)_{0} / \mathbb{Z}_{2}$ through the linear action of $\mathrm{SO}(2, D)_{0}$ on the cone.

Minkowski spacetime is just a (dense) chart with coordinates

$$
x^{\mu}=\frac{\xi^{\mu}}{\xi^{D}+\xi^{D+1}} \quad(\mu=0, \ldots D-1)
$$

of the Dirac manifold, so that the singularity of the inversion and the special conformal transformations is just a coordinate effect. The Poincaré group is the subgroup of $\operatorname{Conf}_{D}$ preserving the auxiliary coordinate $\xi^{D}+\xi^{D+1}$; its generators are $m^{\mu \nu}$ and $p^{\mu}=\frac{1}{2}\left(m^{\mu D}+m^{\mu D+1}\right) \in \operatorname{so}(2, D)(\mu, \nu=0, \ldots D-1)$. Other Minkowski charts are obtained by acting with $\operatorname{Conf}_{D}$ on the Dirac manifold.

Denoting $p_{0}$ the generator of the time translations in the Lie algebra of $\operatorname{Conf}_{D}$, and $k_{0}=I p_{0} I$ the generator of the timelike special conformal transformations, $h_{\text {conf }}:=\frac{1}{2}\left(p_{0}+k_{0}\right)$ is the generator of the compact subgroup $\sim$ $\mathrm{SO}(2) \subset \operatorname{Conf}_{D}$ in the "time" plane $(0-D+1$-plane) of the auxiliary space, in which $I$ is the rotation by $\pi$.

Thus, quantum fields of a QFT with conformal symmetry must be defined as operator-valued distributions on the Dirac manifold. Their restriction to any Minkowski chart are Poincaré covariant Wightman fields in the usual sense.

The discrete spacetime symmetries (parity $P$ and time reflection $T$ ) are not part of the axioms; instead, the CPT theorem applies, stating that while $P$ and $T$ may not be separate symmetries, there is an antiunitary operator $\Theta$ preserving the vacuum vector and acting on the fields like the combination of $P, T$, and a charge conjugation $C$.

Conformal transformations may take pairs of points which are timelike separated in a Minkowski chart to pairs of points at spacelike separation. In other words: the distinction between "spacelike" and "timelike" is Poincaré invariant, but not conformally invariant. As a consequence, causal commutativity of fields at spacelike distance implies causal commutativity also at timelike distance, and the support of the commutator is constrained to the null cone.

This conclusion is avoided if one admits projective representations of the conformal group, such as they occur, e.g., for the massless Klein-Gordon field in odd spacetime dimensions $D$ which has half-integer scaling dimension $d=\frac{D-2}{2}$. 
In these cases, fields are not defined on the Dirac manifold, but on a suitable covering space thereof [56].

\subsection{Two dimensions}

Conformal QFT in two dimensions is in our focus of interest because it admits a multitude of models which can be rigorously constructed. Most of these models possess (finitely or infinitely) many positive-energy representations, which admit, e.g., case studies of the general theory of superselection sectors as originally formulated for Poincaré covariant QFT in four dimensions (generalizing the univalence superselection rule or the electromagnetic charge superselection rule). This theory in particular defines the statistics (a representation of the permutation group) as an intrinsic feature of a positive-energy representation. In two dimensions, a new feature occurs, due to the disconnectedness of the causal complement of a point in two-dimensional Minkowski spacetime: the statistics in general is a representation of the braid group, related (via the Spin-Statistics theorem) to a much wider range of helicities than the Fermi-Bose alternative in $D=4$.

In contrast to $D>2$ dimensions, the conformal group in two spacetime dimensions (2D) turns out to be infinite-dimensional. In fact, the Dirac manifold in two dimensions can be identified with the product of two "chiral" circles

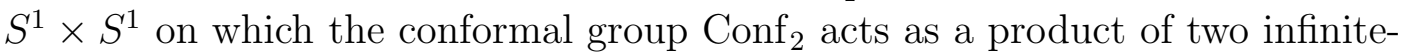
dimensional Lie groups Diff $+\left(S^{1}\right)$ (the orientation-preserving diffeomorphisms).

Namely, one can parameterize the solutions to $\xi \cdot \xi=0$ for $\xi \in \mathbb{R}^{2, D}$ as $\xi=\lambda \cdot(\sin \alpha, \sin \beta, \cos \beta, \cos \alpha)$, so that $[\xi] \leftrightarrow\left(z^{+}=e^{i(\alpha+\beta)}, z^{-}=e^{i(\alpha-\beta)}\right)$ is a bijection between the quotient manifold and the product $S^{1} \times S^{1}$ of two "chiral circles". Moreover, the chiral Minkowski coordinates are

$$
x^{ \pm} \equiv x^{0} \pm x^{1}=\frac{\xi^{0} \pm \xi^{1}}{\xi^{2}+\xi^{3}}=\frac{\sin \alpha \pm \sin \beta}{\cos \beta+\cos \alpha},
$$

such that

$$
z^{ \pm}=\frac{1+i x^{ \pm}}{1-i x^{ \pm}}
$$

This is the Cayley transform $\mathbb{R} \rightarrow S^{1}$ mapping the light ray into the circle (excepting the point $z=-1$ ), whose inverse is the stereographic projection $S^{1} \backslash\{-1\} \rightarrow \mathbb{R}$. Because the Minkowski metric factorizes as $d s^{2}=d x^{+} d x^{-}$, the independent diffeomorphisms of the chiral circles preserve the metric up to a factor.

As it turns out, nontrivial unitary positive-energy representations of the group Diff $+\left(S^{1}\right)$ are necessarily projective representations, and a state invariant under $U_{g}$ for all diffeomorphisms $g$ does not exist. As a consequence, the 
vacuum vector of a conformal QFT in two dimensions is invariant only under the product of the two Möbius groups $\mathrm{SL}(2, \mathbb{R}) / \mathbb{Z}_{2} \sim \mathrm{SU}(1,1) / \mathbb{Z}_{2} \subset \operatorname{Diff}_{+}\left(S^{1}\right)$. This unbroken subgroup of $\operatorname{Conf}_{2}=\operatorname{Diff}_{+}\left(S^{1}\right) \times \operatorname{Diff}_{+}\left(S^{1}\right)$ coincides with the group $\mathrm{SO}(2,2)_{0} / \mathbb{Z}_{2}$ (which is the conformal group $\operatorname{Conf}_{D}$ in $D>2$ dimensions if one puts $D=2$ ).

An important feature of 2D conformal QFT (also related to the factorization of the metric) is the presence of chiral observables, which depend only on $x^{+}$, or on $x^{-}$. These fields are therefore defined on the circle $S^{1}$, into which $\mathbb{R}$ is embedded via the Cayley map. The Möbius group acts by fractional linear transformations $\operatorname{PSL}(2, \mathbb{R})$ on $\mathbb{R} \cup\{\infty\}$ or by fractional linear transformations $\operatorname{PSU}(1,1)$ on $S^{1}$ :

$$
x \mapsto \frac{a x+b}{c x+d}, \quad\left(\begin{array}{ll}
a & b \\
c & d
\end{array}\right) \in \mathrm{SL}(2, \mathbb{R}), \quad z \mapsto \frac{\alpha z+\beta}{\bar{\beta} z+\bar{\alpha}}, \quad\left(\begin{array}{ll}
\alpha & \beta \\
\bar{\beta} & \bar{\alpha}
\end{array}\right) \in \mathrm{SU}(1,1) .
$$

The presence of chiral fields is strongly connected with conservation laws. Because a symmetric tensor field that transforms irreducibly under the conformal group is traceless, it has only two independent tensor components $T_{+\cdots+}$ and $T_{-\ldots-}$ (where \pm stands for Lorentz indices $0 \pm 1$ ). If a symmetric tensor current is conserved, the continuity equation implies that these components are chiral fields, i.e., $\partial_{-} T_{+\cdots+}=0$ and $\partial_{+} T_{-\ldots-}=0$. (This simple argument fails for rank 1 , because the current conservation law $\partial_{\mu} J^{\mu}=0$ gives only one equation. But one notes by inspection that the unique conformally covariant two-point function of a conserved current is also dually conserved, hence by the Reeh-Schlieder theorem also the dual current is conserved: $\partial_{\mu} \varepsilon^{\mu \nu} J_{\nu}=0$. Then again, $J_{+}$and $J_{-}$are chiral fields.) Notice also that the conservation of the conformally invariant two-point function fixes the scaling dimension $d$ of a conserved tensor current, to coincide with the tensor rank $r$.

Conversely, every pair of chiral fields of equal dimension constitutes a conserved traceless symmetric tensor current of rank $r=d$ as a two-dimensional tensor field. Thus, chiral fields naturally occur in 2D conformal QFT models whenever this theory has local conservation laws or, by Noether's theorem, continuous symmetries, and they actually represent the local generators of these symmetries.

The most important conserved tensor current is the stress-energy tensor (SET; dimension $=$ rank $=2$ ), which is (by definition) the local generator of the conformal symmetry $\operatorname{Diff}_{+}\left(S^{1}\right) \times \operatorname{Diff}_{+}\left(S^{1}\right)$ itself. The Lüscher-Mack theorem [57] fixes the self-commutator of its chiral components up to the "central charge" $c$, which can be regarded as the coefficient of the unique central extension of the Lie algebra of the diffeomorphism group of the circle. This quantity is a distinctive invariant of the chiral CFT at hand (where a priori, unless the 2D theory is parity symmetric, the two chiral central charges need not to coincide). 
By local commutativity in the two-dimensional Minkowski spacetime, + and - chiral fields ("left- and right-movers") commute with each other, and chiral fields of the same chirality commute at non-coinciding points, so that their commutators are linear combinations of other fields multiplied with (derivatives of) $\delta$-distributions in the chiral variables $x^{ \pm}$.

Any algebraic relations among chiral fields, including their local commutators, are strongly restricted by conformal covariance. Early attempts at classification of chiral CFTs tried to find consistent commutation and operator product relations (" $W$-algebras"); but in this approach it is in general not known how to assess the existence of representations on a Hilbert space. The most prominent cases where this is possible, are the quantizations of the central charge $c$ below 1 [29], and of the "level" $k$ of chiral current algebras [37], which arise precisely due to the unitarity of the vacuum representation. Both these classification results are made possible by the fact that local commutation relations of the stressenergy tensor and of current algebras can be regarded as central extensions of infinite-dimensional Lie algebras (the Virasoro algebra, and affine Kac-Moody algebras, respectively), which are obtained by extending the fields as operatorvalued distributions on $S^{1}$ and Fourier-decomposing the latter. Then, methods of highest-weight representations of Lie algebras can be applied to obtain the mentioned results.

The most basic examples of chiral fields are free fields related to the 2D massless Dirac and Klein-Gordon fields, which can be constructed in a standard way in terms of creation and annihiliation operators on a Fock space:

- The massless 2D Dirac Fermi field decouples into its two chiral components, where "chiral" stands for the projections onto the eigenvalues of the Dirac matrix $\gamma^{5}=\gamma^{0} \gamma^{1}$ which fix the sign of the helicity relative to the momentum of the massless particles states; by virtue of the massless Dirac equation, the chiral components depend only on $x^{+}$or $x^{-}$, respectively. (While this original meaning of the term "chiral" refers to the helicity, we shall below understand it in the sense of "dependence on $x^{+}$or $x^{-}$only".)

- The canonical massless Klein-Gordon field is ill-defined as an operatorvalued distribution on all test functions in $\mathcal{S}\left(\mathbb{R}^{2}\right)$. It is, however, well-defined on test functions that are derivatives of test functions - which is tantamount to considering the "gradient of the Klein-Gordon field" as an operator-valued distribution on all test functions; this gradient field is of course a conserved current. Its chiral components are chiral fields of scaling dimension $d=r=1$.

The stress-energy tensors of these free fields are not free fields themselves, but can be written as Wick products of the free fields. The SET of a real chiral Fermi field $\psi$ has $c=\frac{1}{2}$, that of the chiral current $j$ has $c=1$. A remarkable feature arises here, known as "fermionization": The chiral current, 
which is a free Bose field, is at the same time a neutral Wick product : $\psi^{*} \psi$ : of a complex free Fermi field (= two real free Fermi fields), acting as the local generator of its $U(1)$ gauge symmetry $\psi(x) \mapsto e^{i \alpha(x)} \psi(x)$. Likewise, the SET of the Bose current $T=\pi: j^{2}$ : coincides with the SET of the complex Fermi field, $T=\frac{i}{2}: \psi^{*} \partial \psi-\partial \psi^{*} \psi$ : , so that the same field has two representations in terms of free Bose or free Fermi fields.

(For the more remarkable converse, called "bosonization", i.e., the representation of fermionic fields in terms of bosonic fields, see Sect.5.3.)

Writing "the same field" in the previous exposition means, that all vacuum correlation functions coincide in both representations. But the vacuum Hilbert space of the SET is just a subspace of the Fock space of the current, which is in turn a subspace of the Fock space of the complex chiral Fermi field. Thus, the latter Fock space carries reducible representations of the current and of the SET.

This simple example leads us to the issue of representations.

\subsection{Representations}

The specific algebraic relations defining a QFT in general admit many inequivalent Hilbert space representations. Covariant representations $\pi$ of the field algebra come with a (projective) unitary representation $U_{\pi}$ of $\operatorname{Conf}_{D}$ whose adjoint action on the covariant fields implements the transformation law:

$$
U_{\pi}(g) \pi(\phi(x)) U_{\pi}(g)^{*}=\pi\left(\alpha_{g}(\phi(x))\right)=D(g, x)^{-1} \pi(\phi(g x)) .
$$

A positive-energy representation is a covariant representation of the field algebra in which the generator of the unitary one-parameter group of time translations (the Hamiltonian) has positive spectrum: $P_{0} \geq 0$. This implies that the commuting generators $P_{\mu}$ of the subgroup of translations have joint spectrum in the closed forward lightcone $\left(P \cdot P \geq 0, P_{0} \geq 0\right)$. A vacuum representation has in addition a unique ground state of zero energy, $P_{0} \Omega=0$.

In conformal QFT, positivity of the Hamiltonian $P_{0}$ is equivalent to positivity of the generator $K_{0}=U(I) P_{0} U(I)^{*}$ of the timelike special conformal transformations, and of the "conformal Hamiltonian" $H_{\text {conf }}=\frac{1}{2}\left(P_{0}+K_{0}\right)$. In 2D, positivity of the generators of chiral Möbius transformations $P^{ \pm}=P_{0} \pm P_{1}$, $K^{ \pm}=K_{0} \mp K_{1}$ and $L_{0}^{ \pm}=\frac{1}{2}\left(P^{ \pm}+K^{ \pm}\right)$follows. $L_{0}^{ \pm}$are the generators of the "rotations" of $S^{1}$.

In the free field example above, the Fock space of the complex Fermi field splits into an infinite direct sum of charged positive-energy representations of the current (the neutral representation being the vacuum representation); in 
turn, as a representation of the SET, the vacuum representation of the current decomposes into an infinite direct sum of representations of the $c=1$ Virasoro algebra. The precise decompositions can be read off the "chiral characters": these are the power series $\chi(t)=\operatorname{Tr} \exp \left(-\beta L_{0}\right)$ or $\chi(t, q)=\operatorname{Tr} \exp \left(-\beta L_{0}-\mu Q\right)$ in the variables $t=e^{-\beta}$ and $q=e^{-\mu}$, which yield the multiplicities of the (integer or half-integer) eigenvalues of the conformal Hamiltonian $L_{0}$ and charge operator $Q$ in the respective representation spaces.

The classification of the positive-energy representations of the Virasoro algebra and of affine Kac-Moody algebras [29, 37] is a breakthrough for QFT without precedent, and without analog in $D=4$ : not only are the algebras generated by the stress-enery tensor, resp. by currents of dimension 1, universally fixed by conformal invariance (up to the central charge in the first case, and up to the structure constants of a Lie algebra and the level in the latter case), but also all their positive-energy representations are explicitly known without a perturbative construction. These models stand at the beginning of many remarkable findings in the representation theory of more general chiral CFTs, including modular tensor categories and the Verlinde formula.

Many issues in QFT are of a basically representation theoretic nature; e.g., the vacuum representation of an extension of a given QFT is in general a reducible representation of the latter, and certain data pertaining to this representation can be used to classify extensions. (An "extension" is, broadly speaking, a QFT containing a given QFT with the same stress-energy tensor as generator of the covariance; see Sect.6.2 for more details.) Another issue are QFTs with boundaries, whose boundary conditions can be understood as different representations of a suitable quotient algebra [6], see Sect. 6.5. These topics can be nicely addressed in two-dimensional CFT and give rise to several nontrivial classifications, because the structure of superselection sectors of chiral CFT is well understood.

In particular, a two-dimensional CFT is an extension of the tensor product of a pair of chiral CFTs; but also many chiral CFTs can be constructed as extensions or subtheories of other chiral CFTs (see Sect.6.2).

\subsection{Different axiomatizations}

Representation theoretic issues are best captured in the algebraic formulation of QFT (AQFT), emphasizing QFT as a given algebraic structure that admits many inequivalent Hilbert space representations. The Haag-Kastler axioms of AQFT therefore do not presume the existence of a vacuum vector - its presence is rather a feature of the representation considered.

Apart from this, the main difference to the Wightman axiomatics is that the 
same physical principles are reformulated in terms of local observables rather than quantum fields, thus offering a somewhat broader generality. We shall briefly review this approach in Sect.3, and be more detailed for chiral conformal QFT in Sect.4, where we also present its most important general results.

It follows from these general axioms that the structure of superselection sectors (= positive-energy representations) of completely rational (see Sect.4) models of chiral CFT is captured by a modular $\mathrm{C}^{*}$ tensor category. The latter is therefore the basic tool to describe the algebraic structure of two-dimensional CFT models (as extensions of chiral ones). There is a wealth of abstract mathematical results about braided and modular tensor categories. We shall point out in Sect.6.2 and Sect.6.5 that many of these abstract results have natural algebraic and representation-theoretic counterparts in the setting of chiral and two-dimensional CFT.

This nearly perfect match is the reason why certain popular axiomations of CFT actually "start from the other end", taking a modular tensor category as the initial axiomatic data. This point of view is justified by the above line of argument, but it tends to create the impression that conformal QFT, rather than being a special case of relativistic QFT, were a "world of its own", with all its peculiar features - notably the presence of a braided or modular tensor category - being not only admitted by the general axiomatic frameworks, but being actually consequences of the usual axioms, specialized to 2D and augmented by conformal symmetry.

Especially, modularity of the representation category should not be regarded as an axiom reflecting some fundamental physical principle, since important models, like the $u(1)$ current algebra, do not share this property. As the characterization in 42 shows, it follows from complete rationality which is rather a regularity property than a fundamental feature.

Yet different axiomatisations, e.g., Euclidean CFT or vertex operator algebras (VOA), also capture essential features of rational CFT, without requiring all the features of relativistic QFT. Notably, the Hilbert space axiom is not essential in some approaches, which therefore admit even more classes of models. Euclidean CFT has a direct physical interpretation independent of its possible correspondence to a real-time relativistic CFT, as critical limits of classical lattice systems in two space dimensions (even with experimental verification). The physical interpretation of "the most general VOA" is not known, but with additional assumptions a close tie with relativistic conformal QFT can be established [23]. 


\section{Algebraic QFT}

The algebraic approach to quantum field theory is formulated by the HaagKastler axioms [35]. The localization of local observables is captured not by the use of quantum fields, but rather by specifying "local subalgebras" of a suitable global $\mathrm{C}^{*}$ algebra. To each open spacetime region is associated a local algebra, whose self-adjoint elements are supposed to represent the physical observables that can be measured (or operations that can be performed) in that region:

$$
O \mapsto A(O) .
$$

Unitary exponentials or spectral projections of self-adjoint smeared field operators would generate local algebras, but this kind of construction is not necessarily assumed in the AQFT approach. It is a nontrivial challenge to find criteria to decide whether local algebras come from Wightman fields, and to extract the latter from the former [16].

The principles of covariance and causality are easily formulated: The group of spacetime symmetries acts by automorphisms $\alpha_{g}$ of the global algebra properly transforming its local subalgebras into each other, and the local algebras associated with two spacelike separated regions are mutually commuting subalgebras.

Appropriate spacetime regions in Minkowski spacetime are doublecones $O$ (intersections of past and future lightcones). With respect to the order relation among doublecones by inclusion, the assignment $O \mapsto A(O)$ is a "net" of local algebras. (Since the set of doublecones in the Dirac manifold is only partially ordered, the local algebras constitute only a pre-cosheaf of algebras.)

By postulating the local algebras to be $\mathrm{C}^{*}$ algebras, and the "global" algebra to be the $\mathrm{C}^{*}$-inductive limit of the local algebras on Minkowski spacetime (usually called the quasilocal algebra), it is ensured that there exist Hilbert space representations. Among all Hilbert space representations, one should select those which describe states of physical interest. This accounts for the fact, that quantum field theory - unlike quantum mechanics - admits in general many inequivalent representations, among which positive-energy representations (distinguished by the implementation of time translations by a unitary one-parameter group with positive generator) and thermal equilibrium representations (distinguished by the presence of a KMS state ensuring the appropriate thermodynamic stability and passivity properties) are the most important ones [16]. Our focus in the sequel will be only on positive-energy representations.

We shall describe various ways of specifying local algebras in Sect.5.

One may object that a "net of local algebras" is a structure too abstract for specifying a particular model - in particular, it does not involve an explicit 
specification of a Lagrangian. But recall that a model of Quantum Mechanics is fixed by specifying the set of observables on the Hilbert space (typically all selfadjoint operators) and the Hamiltonian, i.e., the time evolution automorphisms $\alpha_{t}=\operatorname{Ad}_{U(t)}, U(t)=e^{i H t}$. The same is true in QFT: one has to specify the algebras of observables and the relativistic covariance.

It is a crucial fact in this respect that scattering theory can be carried out (at least in positive-energy representations with a mass gap), by constructing multi-particle states and the scattering matrix, using only the net of local algebras and its covariance. Thus, the local algebras "contain" all the information that is needed to provide the interpretation of a model in terms of particles and their interactions. This is in accord with the fact that, in collider experiments, one usually does not measure a particular field strength but rather deposits of "something" in the detector arrays, and the physical interpretation of this "something" as a particle of either kind is imposed by the correlations of signals in different detector cells (naturally interpreted as "particle tracks" ). In Haag-Ruelle scattering theory, the asymptotic dynamics of any local observable applied to the vacuum state (as long as it is not orthogonal to the desired particle state) is sufficient to identify the asymptotic particle states [35].

The specific dynamics of course enters through the specification of the time evolution automorphisms as part of the covariance. Thus, the Lagrangian (if there is any) is hidden as an "inverse scattering problem" in the scattering theory of the net of local algebras.

There is a marked difference to the standard approach (quantization of a classical Lagrangean theory): it is not a priori required that the generators of spacetime symmetries (in particular the Hamiltonian) are integrals over densities (components of the stress-energy tensor) which are some local "functions" of the field observables. In the classical theory, such relations would imply, through the canonical Poisson brackets, the correct infinitesimal transformation laws on the fields. In quantum field theory, on the other hand, canonical commutation relations cannot hold (in general) in a strict sense. Retaining only the "correct infinitesimal transformation laws" is therefore an appropriate substitute for the quantization of a classical Lagrangean formulation. One should keep in mind that classical physics is only a limit of the "true" quantum physics, and there is no reason to believe in a fundamental 1:1 correspondence between the two realms.

The "lack of fields" in the AQFT framework is in fact another strength: while different sets of quantum fields (relatively local w.r.t. each other) may generate the same local algebras, and hence have the same scattering states and the same scattering matrix (thus they belong to the same "Borchers class"), the local algebras they generate are the same, and should be regarded as the invariant 
content of the theory. The actual choice of fields may rather be regarded as an auxiliary device to describe the algebras (analogous to the choice of coordinates for a manifold), which may be very convenient but is not intrinsic to the physical interpretation.

In the application of the AQFT framework to conformal QFT in two dimensions, nets of local algebras are not just assumed to be given (as often in axiomatic approaches), but can be explicitly constructed by a large variety of methods. Some of these models actually do use fields (e.g., free Fermi fields which are bounded operators after smearing, or currents whose unitary Weyl operators are taken as the generators of the local algebras), but manipulations on such elementary constructions give rise to new nets that cannot always be easily described in the language of fields. We shall contrast the usual "fieldtheoretical" construction methods with the algebraic methods in Sect.5.

Finally, it is a mathematical benefit that one may work with bounded operators, with norm given by the $\mathrm{C}^{*}$ structure, and has not to worry about domains of definition. In this setup, it is easy to say what a "representation" is, and the issue of superselection sectors as unitary equivalence classes of positive-energy representations can be addressed. Algebraic quantum field theory is therefore the ideal setup to approach representation-theoretic issues.

Most of the seminal breakthrough achievements in conformal quantum field theory in two spacetime dimensions are of a representation theoretic nature (classification of the central charge and of conformal dimensions at $c<1$ [29], fusion rules [4], coset models and branching rules [32], ...). Indeed, AQFT provides a unifying framework for these insights, that has proven one of the places where the AQFT formulation is most powerful, by the general theory of superselection sectors initiated by Doplicher, Haag and Roberts [25], see Sect. 4.2 .

\section{Algebraic CFT on the circle}

\subsection{Axioms}

We give here the AQFT axioms for a Möbius covariant chiral QFT (chiral CFT) directly in its vacuum representation, as in [13].

As emphasized earlier, in order to be prepared for the study of more general representations, one should rather axiomatize a pre-cosheaf of abstract local algebras on which the Möbius group acts by automorphisms; but one may as well read off this pre-cosheaf from its vacuum representation, by regarding the latter as the defining representation. 
A chiral CFT is thus given by a family of local algebras $A(I)$ on a Hilbert space $H$ where $I$ runs over the proper open intervals of the circle $S^{1}$ into which the real axis $\mathbb{R}$ is embedded via the Cayley transform Eq. (2.7). One may take $A(I)$ to be von Neumann algebras. The fundamental axioms are:

(1) Isotony. If $I_{1} \subset I_{2}$, then

$$
A\left(I_{1}\right) \subset A\left(I_{2}\right)
$$

(2) Locality. If $I_{1} \cap I_{2}=\varnothing$, then

$$
\left[A\left(I_{1}\right), A\left(I_{2}\right)\right]=0
$$

(3) Möbius covariance. There is a unitary representation $U$ of the Möbius group $\operatorname{PSU}(1,1)=\operatorname{PSL}(2, \mathbb{R})$ on $H$ such that for any interval $I$ and $g \in$ $\operatorname{PSU}(1,1)$,

$$
\alpha_{g}(A(I)):=U_{g} \pi_{0}(A(I)) U_{g}^{*}=A(g I) .
$$

(4) Positive energy. The generator $P$ of the one-parameter subgroup of translations in the representation $U$ has positive spectrum.

(5) Vacuum. There is unique (up to a phase) unit vector $\Omega \in H$ which is invariant under the action of $U$, and cyclic for $\bigvee_{I} A(I)$.

As mentioned above, positivity of the chiral Hamiltonian $P$ is equivalent to positivity of the generator $L_{0}$ of the one-parameter subgroup of rotations (the conformal Hamiltonian). By (4) and (5), the spectrum of $L_{0}$ in the vacuum representation is a subset of $\mathbb{N}_{0}$.

The axioms (1)-(5) imply the following properties [13]:

(6) Reeh-Schlieder property. The vector $\Omega$ is cyclic and separating for each local algebra $A(I)$.

(7) Additivity. If $I=\bigcup_{i} I_{i}$, then $A(I)=\bigvee_{i} A\left(I_{i}\right)$.

(8) Haag duality on $S^{1}$. For any proper interval $I$ one has

$$
A(I)=A\left(I^{\prime}\right)^{\prime}
$$

where $I^{\prime}$ is the interior of the complement of $I$ in $S^{1}$.

(9) Bisognano-Wichmann property. The Tomita modular group [69] $\Delta^{i t}$ of $A\left(\mathbb{R}_{+}\right)$with respect to the vector $\Omega$ coincides with $U\left(\delta_{-2 \pi t}\right)$, where $\delta_{t} \in$ $\operatorname{PSL}(2, \mathbb{R})$ is the one-parameter group of dilations.

(Here and below we are freely using the Cayley identification of $\mathbb{R}$ as a subset of $S^{1}$, and $\operatorname{PSU}(1,1)=\operatorname{PSL}(2, \mathbb{R})$. By virtue of Möbius covariance, the analog of (9) is true for every local algebra $A(I)$ and the associated subgroup of "dilations" of $I$.) 
An interesting consequence of (9) is that the modular groups of the local algebras of any three intervals generate the representation $U$ of the Möbius group PSU $(1,1)$, in particular, spacetime symmetries are of modular origin. Conversely, it was shown in [34] that if $M_{i}$ are three commuting von Neumann algebras with a joint cyclic and separating vector $\Omega$, and $M_{i} \subset M_{i+1}^{\prime} \bmod 3$ are halfsided modular inclusions, then the three modular groups generate a positive-energy representation $U$ of $\operatorname{PSU}(1,1)$. From this, one can construct a conformal net in its vacuum representation satisfying the axioms (1)-(5) (and strong additivity (12) below) by identifying $M_{i}$ with the local algebras of three intervals arising by subdividing the circle by removing three points, and their modular groups with the corresponding dilation subgroups of $\operatorname{PSL}(2, \mathbb{R})$, and using the action of the resulting representation $U$ to consistently define $A(I)$ for general $I$.

(This seems to be an interesting way to construct new chiral CFT models, but ideas to provide triples of commuting von Neumann algebras with the stated properties are scarce if one does not start from a CFT.)

Beyond the basic axioms (1)-(5), one may require further properties that are satisfied for many models.

The presence of a stress-energy tensor is axiomatized as a stronger version of (3):

(10) Diffeomorphism covariance. The representation $U$ of $\operatorname{PSU}(1,1)$ extends to a projective unitary representation of Diff $+\left(S^{1}\right)$ such that for any interval $I$, one has

$$
\operatorname{Ad}_{U(g)} A(I)=A(g I), \quad \text { for } \quad g \in \operatorname{Diff}_{+}\left(S^{1}\right),
$$

and

$$
\operatorname{Ad}_{U(g)} a=a, \quad \text { if } \quad a \in A(I), \operatorname{supp} g \subset I^{\prime} .
$$

By Haag duality (8), the latter property implies that $U(g) \in A(I)$ if supp $g \subset I$, and the subnet generated by such $U(g)$ is called a Virasoro net.

The next two properties express "maximal decoupling" of local observables in intervals at a finite distance, and "maximal interaction" of local observables in touching intervals [55]:

(11) Split property. If $I_{1}$ and $I_{2}$ are two intervals with disjoint closure, then the map $a_{1} \otimes a_{2} \mapsto a_{1} a_{2}$ is an isomorphism of von Neumann algebras

$$
A\left(I_{1}\right) \otimes A\left(I_{2}\right) \simeq A\left(I_{1}\right) \vee A\left(I_{2}\right) .
$$

An equivalent assertion is that states can be independently prepared on the subalgebras $A\left(I_{1}\right)$ and on $A\left(I_{2}\right)$, such that a joint state restricting to the given states on $A\left(I_{i}\right)$ always exists. This feature depends on the energy level density, 
and is known to be true [20] if $e^{-\beta L_{0}}$ is a trace-class operator (in the vacuum representation) for every $\beta>0$. (Such traces, regarded as power series in $t=$ $e^{-\beta}$ whose coefficients give the multiplicities of the spectrum of $L_{0}$, are usually referred to as "characters" and are a very useful tool for the decomposition of reducible representations.)

(12) Strong additivity. Whenever two intervals $I_{1}$ and $I_{2}$ are obtained by removing an interior point from a proper interval $I$, then

$$
A(I)=A\left(I_{1}\right) \vee A\left(I_{2}\right) .
$$

Thinking in terms of quantum fields, (12) may be regarded as a regularity property to the effect that the smearing can be approximated by test functions that vanish at a given point.

In view of (8), strong additivity (12) is equivalent to Haag duality on $\mathbb{R}$, namely for any proper interval $I \subset \mathbb{R}$ one has

$$
A(I)=A\left(I^{c}\right)^{\prime},
$$

where $I^{c}$ is the interior of the complement of $I$ in $\mathbb{R}$.

For the algebra of two intervals $I, J \subset S^{1}$ with disjoint closure, Haag duality will generally fail. The index of the inclusion (in the vacuum representation)

$$
A(I \cup J) \equiv A(I) \vee A(J) \subset A\left((I \cup J)^{\prime}\right)^{\prime}
$$

is called the $\mu$-index (which my be infinite).

A chiral CFT is called "completely rational" [42, 49, 55] if it is split (11), strongly additive (12), and has finite $\mu$-index. A chiral CFT is called "rational" if it possesses only finitely many irreducible superselection sectors, see Sect. 4.2. It was shown in [55] that a split chiral CFT is completely rational if and only if it is rational; in particular, rationality together with split implies strong additivity. Moreover, in a completely rational chiral CFT the braiding is completely non-degenerate, turning the $\mathrm{C}^{*}$ category of superselection sectors (see Sect.4.2) into a modular category [42].

It should be stressed, however, that complete rationality is by no means an obvious feature. One of the most elementary models, the chiral $u(1)$ current algebra, satisfies the split property and strong additivity, but possesses a continuum of charged sectors, and thereby fails to be rational, hence completely rational.

\subsection{Superselection sectors}

A general theory of superselection sectors was originally developped by Doplicher, Haag and Roberts [25] to describe sectors in massive QFT in four spacetime dimensions. With minor modifications in the setup, but big differences in 
the outcome (see below), it has proven to be applicable in two-dimensional and chiral CFT [27].

The crucial assumption on the representations $\pi$ that can be treated by the DHR theory of superselection sectors is that, upon restriction to the causal complement of any doublecone region, $\pi$ is indistinguishable from the vacuum representation up to unitary equivalence:

$$
\left.\left.\pi\right|_{A\left(O^{\prime}\right)} \simeq \pi_{0}\right|_{A\left(O^{\prime}\right)}
$$

The heuristics behind this criterion is the argument that a charge distinguishing two representations can always sit inside the doublecone $O$, which is inaccessible to measurements in its causal complement $O^{\prime}$.

Even for massive theories, this heuristic idea may fail for charges that can only be localized in "topological strings" (narrow cones extending to spacelike infinity) [15], requiring some mild adaptation of the theory. More dramatically, the criterium Eq. (4.11) excludes theories with long-range forces, notably QED, because an electric charge can be detected by measurements in the causal complement due to Gauß' law. An adaptation of the theory to this case has recently been formulated by Buchholz and Roberts [19].

In contrast, the chiral analogue of Eq. (4.11) is automatically satisfied in chiral CFT [18].

Assuming the validity of the criterium Eq. (4.11), positive-energy representations $\pi$ can be described in terms of DHR endomorphisms $\rho$ of the quasi-local algebra [25]. (In the case of CFT on $S^{1}$, where the set of intervals is only partially ordered, one may restrict the CFT to a net on $\mathbb{R} \subset S^{1}$. Otherwise, the definition of DHR endomorphisms is slightly more involved, invoking the pre-cosheaf structure.) Up to unitary equivalence, one has

$$
\pi=\pi_{0} \circ \rho
$$

where $\pi_{0}$ is the (defining) vacuum representation.

The DHR endomorphisms are "localized" in some region, meaning that their action is trivial on the subalgebras of local observables at spacelike distance of that region, and "transportable", meaning that that region can be chosen arbitrarily. The first property is consistent with the idea that $\rho$ arises by conjugation with some localized charged field operator. The second is (a consequence of) covariance, and is consistent with the idea that the total charge does not depend on the localization of the charged field operator. But it should be stressed that these properties are derived without assuming the existence of such a field operator, and are entirely intrinsic in terms of the observables.

Equivalence, direct sums and subrepresentations of positive-energy representations can be formulated directly for the corresponding DHR endomorphisms, 
in terms of intertwiners, which are local observables satisfying the intertwiner relation $t \rho_{1}(a)=\rho_{2}(a) t$. The crucial insight of [25] is that locality, Haag duality and the localization of DHR endomorphisms imply algebraic properties of the intertwiners, which turn the representation theory into a $\mathrm{C}^{*}$ tensor category (the "DHR category").

The composition of DHR endomorphisms describes a product of representations ("fusion") with the vacuum representation (the identical endomorphism) as the "neutral" element. The fusion product is commutative up to unitary equivalence, implemented by distinguished unitary intertwiners ("statistics operators") [25, 27]. The statistics operators turn the DHR category into a braided tensor category. In particular, each irreducible sector can be assigned a "statistics phase" $\kappa_{\rho}$ and a "statistical dimension" $\operatorname{dim}(\rho)$.

In four dimensions, the braiding is in fact a permutation symmetry. As a consequence, $\kappa_{\rho}= \pm 1$ and $\operatorname{dim}(\rho) \in \mathbb{N}$, and these quantum numbers are related to the statistics (and hence the spin) of particles in the associated charge sector [25, II]. In chiral CFT, the conformal spin-statistics theorem [33] relates the statistics phase with the conformal spin, namely the value of the unitary representative $U(2 \pi)$ of the full rotation of $S^{1}$, and hence the spectrum of the generator $L_{0}(\bmod \mathbb{Z})$.

In a large class of chiral CFT models (the completely rational ones, see Sect.4.1), the braiding is in fact maximally non-degenerate, so that the DHR category is even a modular $\mathrm{C}^{*}$ tensor category [42], and the $\operatorname{sum} \sum_{\rho} \operatorname{dim}(\rho)^{2}$ over all irreducible sectors equals the $\mu$-index, see Sect.4.1.

The latter is a very interesting result, quantitatively relating the existence of nontrivial superselection sectors to a quantity that can be "measured" in the vacuum representation.

The large variety of available models opens the door to model studies of general concepts, exploring the range of possibilities admitted by the general principles of local quantum field theory. Although these methods are presently limited to two dimensional conformal QFT, viewing them in the context of general QFT may be instructive for the construction of more realistic models in four-dimensional spacetime. We shall discuss some of these issues in Sect.6.

\section{Chiral model constructions}

\subsection{Free fields}

The most elementary constructions of chiral models are, as always, free fields. As the conformal scaling dimension $h$ specifies the Möbius invariant two-point 
function $\propto(-i /(x-y-i \varepsilon))^{-2 h}$, and the two-point function completely determines a free field, the only "choice" is a scaling dimension $h$ which must be positive in order that the two-point function is a positive-definite scalar product. The two-point function is local iff $h \in \mathbb{N}$, and it is anti-local iff $h \in \frac{1}{2}+\mathbb{N}_{0}$.

The case $h=\frac{1}{2}$ is identical with the chiral component of a massless Majorana resp. Dirac field, which are real resp. complex chiral Fermi fields. The case $h=1$ is identical with the "chiral derivative of the massless Klein-Gordon field", called the free current, see Sect.2.2.

In the AQFT framework, one would rather define the chiral free Fermi field as a CAR algebra over $L^{2}\left(S^{1}\right)$ with the vacuum state $\omega(\psi(f) \psi(g))=\left(\bar{f}, \Pi_{+} g\right)$ specified by the projection $\Pi_{+}$onto the positive-frequency part; and the chiral free current can be defined by the CCR algebra over $C\left(S^{1}\right)$ with symplectic form $\sim i \int\left(f^{\prime} g-f g^{\prime}\right)$ with the vacuum state given by a Gaussian $\omega(W(f))=e^{-\frac{1}{4}\|f\|_{C}^{2}}$ with a suitable inner product of the complexified symplectic space to guarantee positive energy. Local subalgebras are specified by specifying the support of the functions $f$.

\subsection{Wick products and Fermionization}

Quadratic Wick products of free fields are well-defined by standard quantum field theory methods.

In particular, the stress-energy tensor of the free Fermi field is $T=\frac{i}{2}: \psi \partial \psi$ : and the stress-energy tensor of a free current is $T=\pi: j^{2}$, giving the central charge $c=\frac{1}{2}$ and $c=1$, respectively.

"Fermionization" is the remarkable feature that the bosonic free current can be represented as the neutral Wick product $: \psi^{*} \psi$ : of a complex free Fermi field. The bosonic current is therefore defined on the fermionic Fock space, which as a representation splits into an infinite direct sum of charged representations with integer charge. The bosonic stress-energy tensor $\pi: j^{2}$ : coincides with the fermionic stress-energy tensor $\frac{i}{2}: \psi^{*} \partial \psi-\partial \psi^{*} \psi:$.

By way of generalization, one obtains "nonabelian currents" associated with a (semi-simple) Lie algebra $\mathfrak{g}$ by the "quark model construction": choosing an $n$ dimensional real or complex matrix representation $\tau^{a}=\pi\left(X^{a}\right)$ of the generators $X^{a}$ of $g$, one defines the currents as quadratic Wick products of $n$ real or complex free Fermi fields: $j^{a} \sim \tau_{i j}^{a}: \psi_{i} \psi_{j}$ : resp. $j^{a} \sim \tau_{i j}^{a}: \psi_{i}^{*} \psi_{j}$ :

Their commutation relations can be viewed as central extensions of the Lie algebra $L \mathfrak{g}$ of the loop group $L G$, i.e., the $\mathfrak{g}$-valued functions on $S^{1}$; the central term is universal up to a factor, called the level $k$, and the level in the given construction is a function of the Lie algebra $\mathfrak{g}$ and its representation $\pi$. 
The resulting currents $j^{a}\left(f_{a}\right)$ act as infinitesimal generators of gauge transformations $\psi(x) \mapsto e^{-i f_{a}(x) X^{a}} \psi(x)$.

Given a nonabelian current algebra, its stress-energy tensor is given by the Sugawara construction, $T_{S} \sim h_{a b}: j^{a} j^{b}$ : where $h_{a b}$ is the invariant Killing metric, and the normalization factor is determined by the Lie algebra $\mathfrak{g}$. It should be noted that if the currents are obtained by the "quark model construction" on a fermionic Fock space, the Sugawara stress-energy tensor for the currents will in general not coincide with the fermionic stress-energy tensor (see Sect.5.6).

This construction has an analogue in the AQFT framework [61, 73. Let $G$ be a compact Lie group with a unitary representation $\pi$ on $\mathbb{C}^{N}$. One notes that the gauge transformations $\alpha_{g}: \psi(x) \rightarrow \pi\left(g(x)^{-1}\right) \psi(x)$ for $G$-valued functions $g$ on $S^{1}$, i.e., $g \in L G$, are automorphisms of the CAR algebra of $N$ Fermi fields. The criterium for implementability by unitary operators $W(g)$ in the GNS-Hilbert space of the vacuum state (i.e., the Fock space) can be verified to be fulfilled. It follows that the unitaries $W(g)$ define a projective representation of the loop group $L G$. The cohomology class of the cocycle of this representation can be identified with the level, such that, up to a coboundary, $W(g)=\exp i j^{a}\left(f_{a}\right)$ if $g(x)=\exp i f_{a}(x) X^{a}$.

Similarly, the orientation-preserving diffeomorphisms of $S^{1}$ are automorphisms of the CAR algebra via $\psi(f) \mapsto \psi\left(\gamma^{\prime \frac{1}{2}} f \circ \gamma\right)$. Again, these are implemented by unitaries $V(\gamma)$, giving rise to a projective representation of Diff $\left(S^{1}\right)$ on the Fock space. This representation is related to the fermionic stress-energy tensor by $V(\gamma)=\exp i T_{F}(\delta)$ (again, up to a cocycle) if $\delta$ is an infinitesimal diffeomorphism and $\gamma=\exp (\delta)$.

On the other hand, for $\gamma \in \operatorname{Diff}_{+}\left(S^{1}\right)$, the map $g \mapsto g \circ \gamma$ is an automorphism $\alpha_{\gamma}$ of the loop group $L G$. Hence, for any projective representation $\pi$ of $L G$, one obtains another projective representation $\pi \circ \alpha_{\gamma}$. It turns out that this representation is unitarily equivalent to $\pi$, i.e., $\alpha_{\gamma}$ are unitarily implemented by operators $V(\gamma)$ giving rise to a projective representation of $\operatorname{Diff}_{+}\left(S^{1}\right)$ on the representation space of $\pi$. This is the AQFT analogue of the Sugawara construction.

One might object that these theories are just subtheories of free QFT. However, the Sugawara stress-energy tensor of these theories is different from the free-field stress-energy tensor, indicating that the dynamics is different. Accordingly, the current algebras possess many positive-energy representations that do not arise by restriction of free-field representations. The study of the representation theory of chiral CFT models has been in the focus of interest for three decades, and has revealed a host of fascinating connections, including modular invariance of characters [72] and $A-D-E$ classifications [21]. 


\subsection{Bosonization}

A much less-to-be-expected "converse" of fermionization is bosonization. It goes back to Mandelstam's vertex operator construction of free Fermi fields as the exponential of the (non-existing) massless scalar field, where the latter is to be written as an integral over the chiral current. Clearly, this integral makes the construction highly nonlocal. Frenkel and Kac [28] rediscovered this (formal) construction in a clean mathematical setup in terms of well-defined infinite normal ordered exponentials of the Fourier modes of the current on $S^{1}$ on the vacuum Fock space of the current, times a quantum-mechanical factor for the zero mode. The latter factor requires to extend the Hilbert space by a factor $L^{2}\left(S^{1}\right)$ on which the total charge operator acts like a rotation with discrete spectrum. The construction therefore selects a discrete one-dimensional lattice from the continuum of charges (superselection sectors) of the $u(1)$ current.

By exponentiating several $u(1)$ currents with coefficients that take values in an even higher-dimensional lattice, one can construct new local fields in a similar way. Some of these theories coincide with nonabelian current algebras, as in Sect.5.2, at level $k=1$, where the original $u(1)$ currents are the currents for the Cartan subalgebra.

In the AQFT framework, the analogous construction is understood as a crossed product of the Weyl algebra corresponding to the abelian current algebra by a lattice subgroup of the continuous group of DHR automorphisms [18, 68]. This amounts to an extension of the quasilocal algebra by charged intertwiners ("fields") whose charges take value in the lattice. The commutation relations of the fields are determined by the statistics operators of the DHR automorphisms, which turn out to be just complex phases. The extension by the new fields is a local extension if and only if all phases are $=1$, which is precisely the condition that the lattice is even.

A lattice extension corresponding to the "moonshine" vertex operator algebra has been constructed using the 24-dimensional Leech lattice [41. Its central charge is $c=24$, its $\mu$-index is 1 (i.e., it has no nontrivial superselection sectors), its vacuum character is the modular invariant $J$-function and its automorphism group is the Monster group.

\subsection{Orbifold constructions}

One can always descend from a chiral CFT with a compact gauge group of inner symmetries to the gauge-invariant subtheory. There are many examples with finite gauge groups; but one may as well consider the global symmetry of the Lie group $G$ on a current algebra associated with its Lie algebra $\mathfrak{g}$. The fixed 
point subalgebra contains the Sugawara stress-energy tensor, but is in general larger, with very few exceptions.

The formulation as fixed points under the action of a gauge group by automorphisms of the net of local algebras is the same in the AQFT framework.

\subsection{Simple current extensions}

The positive-energy representations (sectors) do in general not form a group under the fusion product; in particular, there is no inverse but only a conjugate such that the product with the conjugate contains the trivial (=vacuum) sector. Sectors which have an inverse (i.e., the fusion product is the trivial sector), are called simple sectors (or "simple currents" in some communities). In the DHR theory, simple sectors are given by DHR automorphisms, rather than endomorphisms.

Simple current extensions are extensions of a chiral CFT by local fields that carry the charge of simple sectors. By the Spin-Statistics Theorem, such fields can only be local if they carry integer spin, hence the statistics phases must be $=1$. In order to define the extensions, one has to specify consistent algebraic relations between the new fields and the old fields, and among the new fields. The problem can often be reduced to a control of the representation category of the extended theory, in terms of that of the original theory.

The simple sectors with trivial statistics phases form a group under the fusion product. In the AQFT framework, simple current extensions can be defined as crossed products of the original net by the action of this group, in much the same way as the lattice extension of Weyl algebras in Sect.5.3.

\subsection{The coset construction}

If a local QFT contains a sub-theory, the "coset QFT" is generated by all local fields of the larger theory that commute with the sub-theory. Thinking of the latter as generators of a symmetry (currents as generators of gauge transformations, the SET as generator of diffeomorphisms), the coset fields are invariant under that symmetry. Specifically, an inclusion $\mathfrak{h} \subset \mathfrak{g}$ of Lie algebras induces an inclusion of the current algebra chiral CFTs. Both these CFTs have their own Sugawara stress-energy tensor. Both stress-energy tensors are generators of the same (universal) diffeomorphism transformations of the $h$-currents, which means that their difference commutes with these currents. One obtains the "coset stress-energy tensor" $T_{\mathfrak{g}}-T_{\mathfrak{h}}$ with central charge $c_{\mathfrak{g}}(k)-c_{\mathfrak{h}}\left(k^{\prime}\right)$ [32].

Since the coset stress-energy tensor is different from the pair of given stressenergy tensors, the coset theory has its own dynamics. In particular, in this way 
all stress-energy tensors with central charge $c<1$ have been constructed [32], thus rounding off the classification of admissible values of $c<1$ "by exclusion of the continuum" 29] by an existence result for the remaining discrete set.

The coset construction allows to construct "new models from old models", and is by no means restricted to current algebras associated with Lie subalgebras $\mathfrak{h} \subset \mathfrak{g}$.

In the AQFT framework, the coset construction is given by a relative commutant of local algebras, namely, if $A(I) \subset B(I)$ are the local algebras of a chiral CFT and a subtheory, then the local algebras of the coset theory are

$$
C(I):=A(I)^{\prime} \cap B(I) .
$$

Some of the Virasoro algebra theories with $c<1$ admit extensions by further local fields, without increasing the central charge. A complete classification has been obtained by AQFT methods (building on the earlier classification of modular invariant matrices), see Sect.6.2

\section{Algebraic constructions (not only conformal)}

To construct a model in the AQFT framework, one has to specify the local algebras along with the automorphic action of the spacetime symmetries, and one has to provide a vacuum representation with the appropriate spectral properties.

Starting from a given model, one possibility is to extend it by enlarging the local algebras. In order to exclude trivial "extensions by tensor products", one would require the extension to be irreducible, i.e., to have trivial relative commutant. The extended model will in general require a larger Hilbert space.

Another possibility is to deform the local algebras on the same Hilbert space, while preserving the local commutativity and covariance. For a more extensive review of this approach, see [47].

A third idea is "holographic" in the sense that the spacetime association of observables in a given net of local algebras is radically reorganized, such that, e.g., the quantum observables of a chiral CFT are re-arranged to become the observables of a two-dimensional model.

We shall present examples of these new construction ideas in the sequel.

\subsection{Superselection sectors and symmetry}

Unbroken inner symmetries give rise to superselection sectors. 
Let $G$ be a compact global gauge group with an action by automorphisms $\gamma_{g}$ $(g \in G)$ on a local QFT $B$ such that $\gamma_{g}$ preserve the local algebras and commute with the spacetime covariance. Let furthermore the vacuum state $\omega_{B}$ of $B$ be invariant under $\gamma_{g}$, i.e., the vacuum does not break the symmetry, so that the gauge transformations are implemented by a unitary representation of $G$ on the vacuum Hilbert space $H_{B}$.

The net of fixed-point subalgebras $A(O)=B(O)^{G}$ inherits the local structure and the spacetime covariance, and $H_{B}$ carries a reducible positive-energy representation of $A$. It decomposes into subspaces $H_{\pi}$ generated from the vacuum vector by elements of $B$ transforming in some representation $\pi$ of $G$, and each subspace $H_{\pi}$ is invariant under the invariants $A$. In particular, the cyclic subspace $H_{A}$ generated by $A$ from the vacuum vector is a proper subspace of $H_{B}$.

One can reconstruct the full vacuum representation of $B$ from the vacuum state $\omega_{A}$ of $A$, via the GNS construction of the state $\omega_{B}=\omega_{A} \circ \mu$, where $\mu$ is the conditional expectation from $B$ onto $A$ given by the Haar average over the group action.

A breakthrough result by [26], valid in QFT in four-dimensional spacetime, shows that the scenario just described is the generic origin of superselection sectors. It relies on the fact that in spacetime dimension $>3$ (and often also $=$ 3 ), the category of DHR superselection sectors [25] is a permutation symmetric $\mathrm{C}^{*}$ tensor category, and such categories can be identified with the dual of a compact group. It proceeds by reconstructing from the given local net $A$ and its DHR category a (unique up to isomorphism) universal "field algebra" $B$ (which may be graded local) together with an action of a compact gauge group $G$ such that $A=B^{G}$ is the fixed-point subalgebra, and the DHR sectors $\rho$ of $A$ are in 1:1 correspondence with the unitary representations $\pi$ of $G$ with statistical dimension $\operatorname{dim}(\rho)=$ matrix dimension $\operatorname{dim}(\pi)$.

As a consequence of this result, every irreducible extension of $A$ is the intermediate algebra of invariants of $B$ under a subgroup $H \subset G$.

It may appear natural to expect some inner symmetry also to be at the origin of superselection sectors in low-dimensional spacetime. However, there are obstructions due to the fact that the braiding is not a permutation symmetry in low dimensions (which is in turn a consequence of the geometric property that the causal complement of a finite connected region has two connected components). As a consequence, the dimensions $\operatorname{dim}(\rho)$ fail to be integer in general, and a 1:1 association with finite-dimensional representations $\pi$ of some inner symmetry such that $\operatorname{dim}(\rho)=\operatorname{dim}(\pi)$ as before cannot be expected.

Yet, it is possible to associate a (non-unique) weak $\mathrm{C}^{*}$ Hopf algebra [62] with the DHR category of a local QFT $A$, where the non-integrality of dimensions 
enforces the failure of the property $\Delta(1)=1 \otimes 1$ of the coproduct. The "reduced field bundle" $F$ of [27, II] can be interpreted as a nonlocal sector-generating field algebra with an action of a weak $\mathrm{C}^{*}$ Hopf algebra such that the invariants are the observables $A$. An undesired but unavoidable feature of this construction is that the embedding $A \subset F$ is not irreducible, and $F$ contains elements which belong to each of its local algebras.

\subsection{Extensions by Q-systems}

An alternative approach was initiated in [51], by characterizing irreducible covariant extensions $A \subset B$ of a given local QFT $A$. It is assumed that $B$ is relatively local w.r.t. $A$, i.e., observables of $B$ commute with observables of $A$ at spacelike distance, and that there is a conditional expectation $\mu: B \rightarrow A$ taking $B$ onto $A$ and preserving the vacuum state. One does not assume any specific symmetry concept (like group or weak Hopf algebra), but retains only the conditional expectation as a substitute for the Haar average over the action of the gauge group.

It is also assumed that the index of the local subfactors $A(O) \subset B(O)$ (which is independent of $O$ ) is finite, which is automatic if $A$ is completely rational.

This scenario includes simple current extensions as well as orbifold constructions (regarding the full algebra as an extension of the fixed points), and conformal embeddings (local CFTs that share the same stress-energy tensor 65]) as well as coset constructions (regarding $B$ as an extension of $A \otimes C$ if $C$ is the coset model of $A \subset B$ ). But it is more general since it is not required that the extension $B$ of $A$ is itself local.

The main result is that every such extension is characterized by a "Qsystem" in the DHR category (or "DHR triple"), and every Q-system allows to reconstruct the extension $B$ in terms of data pertaining solely to $A$ and its DHR category.

A Q-system is a triple, consisting of a DHR endomorphism $\theta$ of $A$ and a pair of intertwiners $w \in \operatorname{Hom}\left(\mathrm{id}_{A}, \theta\right), x \in \operatorname{Hom}\left(\theta, \theta^{2}\right)$ satisfying the relations

$$
w^{*} w=x^{*} x=d \cdot \mathbf{1}, \quad w^{*} x=\theta\left(w^{*}\right) x=\mathbf{1}, \quad x x=\theta(x) x,
$$

where $d^{2}=\operatorname{dim}(\theta)$. In a more abstract category setting, a Q-system is the same thing as a (standard) Frobenius algebra in a $\mathrm{C}^{*}$ tensor category [7], where the category at hand is the DHR category of superselection sectors.

From the data of the Q-system, the net $B$ is reconstructed as an extension of $A$. It comes equipped with a local structure $A(O) \subset B(O)$ and covariance, and with a conditional expectation $\mu$ respecting the local structure and commuting 
with the covariance. The GNS representation of the state

$$
\omega_{B}=\omega_{A} \circ \mu
$$

as in Sect.6.1 gives the vacuum representation of $B$, which - as a representation of $A$ - is equivalent to the DHR representation $\theta$. To every subsector $\rho \prec \theta$ corresponds a generator $\psi_{\rho}$ of $B$ that interpolates the vacuum subspace to the subspace carrying the representation $\rho$, and that implements $\rho$ "in the average", namely

$$
\rho(a)=\mu\left(\psi_{\rho} a \psi_{\rho}^{*}\right) .
$$

The algebraic relations among the "charged fields" $\psi_{\rho}$ are encoded in the intertwiners $w$ and $x$ that specify the Q-system.

The extension is by construction relatively local w.r.t. $A$, and it is local if and only if the intertwiner $x$ satisfies the condition $\varepsilon_{\theta, \theta} x=x$, where $\varepsilon_{\theta, \theta}$ is the statistics operator.

Thus, exhibiting Q-systems by solving their defining algebraic relations within the DHR category of the given subtheory, amounts to a construction of (relatively local or local) extensions. This is a finite-dimensional problem in rational theories, since for irreducible extensions, one can show that the multiplicity of every sector $\rho \prec \theta$ is bounded by its dimension; hence there are only finitely many "a priori candidates" for $\theta$, and the intertwiner spaces, where $w$ and $x$ take values, are also finite-dimensional. Thus, even lacking more inspired methods (see below), Q-systems in a given rational $\mathrm{C}^{*}$ tensor category can in principle be classified "by brute force".

A very useful fact is that possibly nonlocal chiral extensions $A \subset B$ induce local two-dimensional extensions $A \otimes A \subset B_{2}$, that are "CFT realizations of modular matrices". The original construction [63] uses " $\alpha$-induction", and was recently recognized [5] to coincide, in terms of the corresponding Q-systems, with the "full centre", which is a most interesting concept in braided tensor categories [31, 45].

Complete classifications of local extensions have been achieved [39, 40] for the chiral and two-dimensional Virasoro nets with $c<1$. The authors have exploited the fact that the Virasoro nets with $c<1$ are completely rational, hence their DHR categories are modular (the braiding is non-degenerate). In this case, one can associate a modular invariant matrix with every chiral Qsystem [10, 11], and these matrices have been classified before [21]. Since the DHR representation $\theta$ of the underlying chiral Q-system can be read off the modular invariant matrix, the number of candidates for $\theta$ is drastically reduced. The authors then show existence and uniqueness of the Q-system for each $\theta$ (in the chiral case) by using more abstract existence and uniqueness results of [43], 
and they classify the Q-systems for a given $\theta$ by a certain second cohomology [36] in the two-dimensional case.

It turns out that all local chiral extensions are: an infinite series of $\mathbb{Z}_{2}$ simple current extensions, and four exceptional extensions labelled $\left(A_{10}, E_{6}\right)$, $\left(E_{6}, A_{12}\right),\left(A_{28}, E_{8}\right)$, and $\left(E_{8}, A_{30}\right)$ according to the $A$-D-E classification [21] of their modular invariants. Of these, three have been identified with coset extensions using current algebras [9], except $\left(A_{28}, E_{8}\right)$ which occurs at $c=\frac{144}{145}$. This one was later identified with the "mirror" of a coset extension, where the mirror construction [74] is an operation on Q-systems relating a Q-system in $A$ to a $\mathrm{Q}$-system in $C$ if $A$ and $C$ are each other's relative commutants (coset models) within some common extension $B$.

\subsection{Borchers triples and deformation methods}

A net of local algebras $A(O)$ in any dimension can be constructed from a "Borchers triple" (or "causal triple"). A Borchers triple consists of a von Neumann algebra $M \subset B(H)$ with a cyclic and separating vector $\Omega \in H$, and a unitary positive-energy representation $U$ of the proper orthochronous Poincaré group $P_{+}^{\uparrow}$ on $H$ for which $\Omega$ is the unique invariant ground state. It is required that

$$
\begin{array}{ccc}
U(\lambda) M U(\lambda)^{*} \subset M & \text { whenever } & \lambda W_{0} \subset W_{0} \\
U(\lambda) M U(\lambda)^{*} \subset M^{\prime} & \text { whenever } & \lambda W_{0} \subset W_{0}^{\prime},
\end{array}
$$

where $\lambda$ stands for $(\underline{a}, \Lambda) \in P_{+}^{\uparrow}, W_{0}=\left\{\underline{x} \in \mathbb{R}^{1, D-1}: x^{1}>\left|x^{0}\right|\right\}$ is the standard wedge region of Minkowski spacetime, and $W_{0}^{\prime}=\left\{\underline{x}: x^{1}<-\left|x^{0}\right|\right\}$ its causal complement.

Clearly, in every QFT, the algebra $M=A\left(W_{0}\right)$ of the standard wedge in the vacuum representation gives a Borchers triple, by virtue of covariance and locality.

Conversely, every Borchers triple defines a net by a simple construction. The construction proceeds by defining $A\left(W_{0}\right):=M$ and $A\left(\lambda W_{0}\right):=U(\lambda) M U(\lambda)^{*}$ for $\lambda \in P_{+}^{\uparrow}$. Then one defines

$$
A(O):=\bigcap_{W \supset O} A(W)
$$

where the intersection runs over all Poincaré transforms $W=\lambda W_{0}$ of the standard wedge which contain $O$. The assumptions Eq. (6.4) ensure that the algebras $A(W)$ are well-defined, and that the net $A(O)$ is local and covariant. (Unfortunately, the algebras $A(O)$ may fail to satisfy the Reeh-Schlieder property $(A(O) \Omega$ dense in $H)$, and may be as small as $\mathbb{C} \cdot \mathbf{1}$.) 
For Borchers triples in two dimensions, the second condition of Eq. (6.4) is obsolete (because there are no such $\lambda \in P_{+}^{\uparrow}$ ). Moreover, it is sufficient to have a positive-energy representation of the translations only: one can then use Tomita's Modular Theory to reconstruct also the representation of the Lorentz group including the CPT conjugation. Namely, Borchers [12] has discovered that the inclusions $U(a) M U(a)^{*} M \subset M$ for $a \in W_{0}$ are half-sided modular, and the modular group $\Delta^{i t}$ and the modular conjugation $J$ of $(M, \Omega)$ satisfy the same commutation relations with the translations $U(a)$ as the Lorentz group $U\left(\Lambda_{-2 \pi t}\right)$ and the CPT conjugation $U(\Theta)$, so one can define $U\left(\Lambda_{t}\right):=\Delta^{-i t / 2 \pi}$ and $U(\Theta):=J$, and the second of Eq. (6.4) is automatic for $\lambda$ involving the conjugation.

(In an effort to generalize this powerful result to $D=4$, Kähler and Wiesbrock [38] have given a characterisation of the "relative modular position" of several von Neumann algebras with a joint cyclic and separating vector, such that their modular groups generate the four-dimensional Poincaré group.)

Exhibiting Borchers triples amounts to the construction of a QFT, with any prescribed particle content specified by the representation $U$.

The difficulty is, of course, to find algebras $M$ satisfying the assumptions, and to find criteria such that the intersections $A(O)$ are large enough. This is easy for free theories, where $M$ is generated by the Weyl operators of the free field smeared within $W_{0}$. Using Modular Theory, one can also define the wedge algebras for free fields associated with Wigner's massless "infinite-spin" representation [14, but the last step defining $A(O)$ for doublecones fails [44, 50]: the intersections of algebras turn out to be trivial unless $O$ contains an (arbitrarily narrow) infinite spacelike cone. Indeed, "string-local" fields associated with the infinite-spin representation have been constructed in [58].

The prevailing ideas for finding more examples proceed by deformations $\widetilde{M}$ of free-field (or any other given) algebras $M=A\left(W_{0}\right)$, so as to produce deformed nets $\widetilde{A}(O)$. They are particularly successful in two dimensions:

Lechner [46] has constructed integrable massive models with factorizing scattering matrix by deformations of the canonical commutation relations (Zamolodchikov-Faddeev algebra). The input in this approach is a scattering function (in the rapidity variable), subject to restrictive analyticity conditions. The question whether the local algebras $A(O)$ are sufficiently large can be answered by a regularity criterium on the scattering function [46]. In the affirmative case, the scattering matrix of the resulting deformed local quantum field theory factorizes into two-particle scattering matrices given by the input scattering function.

Another deformation method uses "warped convolutions" [17, 1, 2]. These can be regarded as "momentum dependent translations" of the elements of wedge algebras where the spectrum of the momentum ensures that wedge- 
locality is preserved. This deformation violates Lorentz invariance in more than two dimensions.

Chiral conformal QFT can be used as a starting point to construct both massive and massless new two-dimensional QFT models through "Longo-Witten endomorphisms", as follows.

If $A$ is a chiral CFT net, then $\left(M=A\left(\mathbb{R}_{+}\right), \Omega, T=\left.U\right|_{\mathbb{R}}\right)$ is a chiral Borchers triple, namely, the translations $T(a)$ with $a>0$ satisfy $T(a) M T(a)^{*} \subset M$ for $a>0$. In order to get a two-dimensional Borchers triple, one has to extend $T$ to a unitary representation $T_{2}$ of the two-dimensional translations $\mathbb{R}^{2}$ with positive energy, such that $T(\underline{a}) M T(\underline{a})^{*} \subset M$ for $\underline{a} \subset W_{0}$.

A Longo-Witten endomorphism [54] is an endomorphism of $M$ of the form $\operatorname{Ad}_{V}$, where $V$ is a unitary operator commuting with $T$ and preserving $\Omega$. Therefore, every one-parameter semigroup of Longo-Witten endomorphisms $V(b)=e^{-i b \widetilde{P}}(b>0)$ with positive generator $\widetilde{P}$ gives rise to a two-dimensional Borchers triple by putting $T_{2}(t, x)=T(t+x) V(t-x)$.

For the net $A$ generated by the free chiral current, semigroups of LongoWitten endomorphisms that arise by second quantization of a unitary semigroup $V_{1}(b)$ on the one-particle subspace, have been classified [54], namely $V_{1}(b)$ turn out to be "singular symmetric inner functions" $\varphi_{b}\left(P_{1}\right)$ of the chiral one-particle momentum operator $P_{1}$. (A symmetric inner function is the boundary limit of an analytic function on the upper complex halfplane with $|\varphi(p)|=1$ and $\varphi(-p)=\overline{\varphi(p)}$ for almost all $p \in \mathbb{R}$. These conditions precisely ensure that the unitary $V=\varphi\left(P_{1}\right)$ implements a Longo-Witten endomorphism at the oneparticle level. Symmetric inner functions are closely related to the admissible scattering functions in Lechner's massive deformations [46], but the physical significance of this relation remains to be explored.) To get a one-parameter semigroup, $\varphi_{b}(p)=e^{i b f(p)}$ must be singular, i.e., it must not have zeros in $\mathbb{C}_{+}$.

The corresponding generator $\widetilde{P}_{1}=-f\left(P_{1}\right)$ is positive iff $f(p)=-m^{2} p^{-1}$ for some $m^{2}>0$. The resulting two-dimensional QFT with chiral translations $P_{1}$ and $\widetilde{P}_{1}$ is just the free massive scalar field (defined on the Hilbert space of the free bosonic current), because $\underline{P}_{1}^{2}=P_{1}^{+} P_{1}^{-}=P_{1} \widetilde{P}_{1}=m^{2} \cdot \mathbf{1}$. This is the converse of the well-known fact that the restriction of the massive free field to a light ray is the conformal free current.

The interesting challenge is to find other one-parameter semigroups of LongoWitten endomorphisms with positive generator that are not of this simple (second-quantized) form.

Pursuing this idea, Tanimoto [70 constructed a large class two-dimensional (massless) Borchers triples from a chiral Borchers triple $\left(M=A\left(\mathbb{R}_{+}\right), \Omega, T=\right.$ $\left.\left.U\right|_{\mathbb{R}}\right)$. These constructions proceed by deformations of the two-dimensional 
tensor product theory $A \otimes A$ whose Borchers triple is $\left(A\left(W_{0}\right)=M \otimes M^{\prime}, \Omega \otimes\right.$ $\left.\Omega, T_{2}\right)$ with $T_{2}(\underline{a})=T\left(a^{+}\right) \otimes T\left(a^{-}\right)$. The deformations act by conjugations on the subalgebras $M \otimes \mathbf{1}$ and $\mathbf{1} \otimes M^{\prime}$ with different unitary operators:

$$
M_{V}=V(M \otimes \mathbf{1}) V^{*} \vee V^{*}\left(\mathbf{1} \otimes M^{\prime}\right) V .
$$

With suitable conditions on the unitary operator $V$, the triple $\left(M_{V}, \Omega \otimes \Omega, T_{2}\right)$ is a deformed two-dimensional Borchers triple, whose energy-momentum spectrum is unchanged. With an appropriate adaptation of scattering theory to the massless situation, the nontrivial scattering matrix of this deformed QFT coincides with the square $V^{2}$ of the deformation unitary.

Depending on the choice of $V$ as a function of the chiral momentum operators, one obtains models that are equivalent, respectively [48, 70], to a massless version of the integrable deformations by a scattering function as in [46], or to the deformations by warped convolutions [17]. Yet different choices of $V$ of the form $e^{i \kappa Q \otimes Q}$, where $Q$ is the generator of an inner symmetry of the chiral theory and $\kappa$ a real deformation parameter, give new classes of deformed models [70, 8].

Starting instead from a two-dimensional (massive) Borchers triple $(M=$ $\left.A\left(W_{0}\right), \Omega, T=\left.U\right|_{\mathbb{R}^{2}}\right)$, the tensor product $\left(M \otimes M, \Omega \otimes \Omega, T^{(2)}\right)$ with $T^{(2)}(\underline{a})=$ $T(\underline{a}) \otimes T(\underline{a})$ is the Borchers triple of two uncoupled identical QFT models. A deformation interaction is introduced by deforming the wedge algebra:

$$
M_{V}^{(2)}:=V(M \otimes \mathbf{1}) V^{*} \vee V^{*}(\mathbf{1} \otimes M) V,
$$

where $V$ is again of the form $e^{i \kappa Q \otimes Q}$ with a suitable self-adjoint generator $Q$. Depending on this choice, the Reeh-Schlieder property of the local algebras can be established [71].

\subsection{Holographic models}

Let $B$ be a chiral QFT net on the real line. For any pair of intervals $K \subset L$ with non-touching end points, let the intervals $I$ and $J$ be the two connected components of $L \backslash \bar{K}=I \cup J$ such that $I>J$ (elementwise). Let

$$
O=I \times J:=\left\{(t, x) \in \mathbb{R}^{1,1}: t+x \in I, t-x \in J\right\} .
$$

Then $O$ is a doublecone contained in the Minkowski halfspace $\mathbb{R}_{+}^{1,1}=\{(t, x) \in$ $\left.\mathbb{R}^{1,1}: x>0\right\}$.

Defining

$$
B_{+}(O):=B(K)^{\prime} \cap B(L),
$$

one obtains a local net of local algebras on the halfspace, covariant under the diagonal of the direct product of two diffeomorphism groups, acting on $t+x \in \mathbb{R}$ and $t-x \in \mathbb{R}$, respectively. 
This is the prototype of a "holographic" construction, since every local operator of $B$ (a one-dimensional net on $\mathbb{R}$ ) is a local observable of $B_{+}$(a twodimensional net on $\mathbb{R}_{+}^{1,1}$ ), but the localization assigned to it is very different. In the conformal case, this is precisely the algebraic AdS-CFT correspondence [64], where $\mathbb{R}_{+}^{1,1}$ appears as a chart of the two-dimensional Anti-deSitter spacetime.

In order to ensure locality of $B_{+}$, it is actually not necessary that $B$ is local: it is sufficient that $B$ is relatively local w.r.t. a local subnet $A$ on $\mathbb{R}$. In this case, $B_{+}(O)$ contains at least the subalgebra $A_{+}(O):=A(I) \vee A(J)$, but already for $B=A, B_{+}(O)$ is strictly larger than $A_{+}(O)$ whenever $A$ possesses nontrivial DHR sectors.

E.g., if $A$ is a Virasoro net, then the halfspace net with local algebras $A_{+}(O)=A(I) \vee A(J)$ has the obvious physical interpretation as the algebra generated by a two-dimensional stress-energy tensor localized in $O$, whose chiral components $T_{+}=T_{-}$are identified by the boundary condition $\left.T^{01}(t, x)\right|_{x=0}=0$. This condition is just the conservation of energy at the boundary $x=0$.

More generally speaking, the holographic halfspace models Eq. (6.7) are extensions of chiral halfspace CFTs with local algebras $A_{+}(O)$, which arise by means of a boundary condition on two-dimensional chiral fields; and every such extension is of the form Eq. (6.7) (or intermediate between $A_{+}(O)$ and $B_{+}(O)$ ) 52 .

There also exist algebraic prescriptions to "remove the boundary" 53. and to "add a boundary" [22], which allow to pass between extensions $A_{+} \subset B_{+}$on the halfspace and extensions $A_{2} \subset B_{2}$ of CFTs on two-dimensional Minkowski spacetime, where $A_{2}(O)=A(I) \otimes A(J)$ is the local algebra of a pair of independent (although isomorphic) chiral algebras. Under the local isomorphism (in completely rational models) $A(I) \vee A(J) \sim A(I) \otimes A(J)$, these pairs of extensions are locally (but of course not globally) isomorphic.

As discussed in Sect.6.2, we can think of $A \subset B$ as a relatively local chiral extension, described by a Q-system of $A$. Via the holographic construction, this produces a local extension $A_{+} \subset B_{+}$on the halfspace, and by "removing the boundary", one arrives at a local two-dimensional extension $A_{2} \subset B_{2}$. The Q-system for $A_{2} \subset B_{2}$ as a "functional" of the underlying chiral Q-system for $A \subset B$ turns out to be precisely the $\alpha$-induction construction [63] which was discovered without knowing the steps just described. More recently [5], it was also identified with the "full centre" of the chiral Q-system, as defined in [31, 45] in the abstract tensor category setting, providing yet a different line of construction for the same extension $A_{2} \subset B_{2}$. 


\subsection{Phase boundaries}

In contrast to the "hard" boundaries as encountered in the previous subsection (physics is defined only in a halfspace), phase boundaries may separate "different physics" on both sides of the boundary. In two-dimensional conformal QFT, imposing the conservation of energy and momentum at the boundary implies that the two local QFTs on both sides share the same $2 D$ stress-energy tensor, and further boundary conditions may imply more common chiral observables.

The issue is thus to have two possibly different local quantum field theories $B^{L}$ and $B^{R}$, each defined in halfspaces $\mathbb{R}_{L}^{1,1}, \mathbb{R}_{R}^{1,1}$, to be represented on the same Hilbert space such that two requirements are respected: $B^{L}$ and $B^{R}$ share a common subtheory $A$, defined on full Minkowski spacetime $\mathbb{R}^{1,1}$, and all observables satisfy Einstein causality, i.e., any two observables at spacelike distance commute.

Because the stress-energy tensor (contained in $A$ ) generates the full spacetime covariance, it can be used to extend both halfspace nets to the full Minkowski spacetime (with their local operators "on the wrong side of the boundary" not being considered as observables). Thus, one has two full local QFTs with a common subtheory defined on the same Hilbert space, where Einstein causality for the observables is equivalent to $B^{L}$ being "left local" w.r.t. $B^{R}$, i.e., a pair of observables of $B^{L}$ and $B^{R}$ commutes if the former is localized at the spacelike left of the latter.

This algebraic situation has been analysed in [6], see also [7]. It is found that, if the local extensions $A \subset B^{L}$ and $A \subset B^{R}$ are given by their Q-systems, there is a universal extension $A \subset C$ given by the "braided product" of the Qsystems. This extensions contains both $B^{L}$ and $B^{R}$ as intermediate extensions, and $B^{L}$ is left local w.r.t. $B^{R}$. It is universal in the sense that every irreducible joint representation of $B^{L}$ left local w.r.t. $B^{R}$ is a quotient of $C$. Thus, to classify such representations, one has to compute the centre of the universal extension $C$.

As a linear space, the centre of the algebra $C$ is generated by neutral products $\Psi_{\rho}^{L *} \Psi_{\rho}^{R}$ of charged fields from $B^{L}$ and $B^{R}$ (where $\rho=\rho_{1} \otimes \rho_{2}$ is a DHR sector of $A \otimes A$ common to both Q-systems). It is more ambitious to compute the centre as an algebra, in order to determine its minimal projections. This can be achieved [6, 7] if the underlying chiral CFT is completely rational and both extensions are given as full centres ( $\alpha$-induction construction from chiral Q-systems, see Sect.6.2): In this situation, the minimal central projections are in 1:1 correspondence with the irreducible bimodules between the underlying chiral Q-systems. Each minimal projection assigns numerical values to the op-

erators $\Psi_{\rho}^{L *} \Psi_{\rho}^{R}$, and thereby specifies the boundary conditions valid among the charged fields. In some cases, but not always, they become linear relations of 
the form $\Psi_{\rho}^{L}=\omega \Psi_{\rho}^{R}$ with phase factors $\omega$.

As an example, we give the classification for the two-dimensional Ising model, which is originally defined as the continuum limit of a lattice model of two-dimensional Statistical Mechanics at the critical point, but can (via an Osterwalder-Schrader "Wick rotation") be regarded as a relativistic quantum field theory. Its chiral net is given by the Virasoro net $A$ with central charge $c=\frac{1}{2}$. The Ising model is then the unique maximal local two-dimensional extension $B \supset A \otimes A$, which hase two charged fields $\Psi_{\sigma \otimes \sigma}$ (the "order parameter") and $\Psi_{\tau \otimes \tau}$ (the product of two chiral Fermi fields).

One finds three bimodules, hence three boundary conditions, expressed in terms of relations between the charged fields:

$$
\begin{gathered}
\text { (i) } \Psi_{\tau \otimes \tau}^{L}=\Psi_{\tau \otimes \tau}^{R}, \quad \Psi_{\sigma \otimes \sigma}^{L}=\Psi_{\sigma \otimes \sigma}^{R} ; \\
\text { (ii) } \Psi_{\tau \otimes \tau}^{L}=\Psi_{\tau \otimes \tau}^{R}, \quad \Psi_{\sigma \otimes \sigma}^{L}=-\Psi_{\sigma \otimes \sigma}^{R} ; \\
\text { (iii) } \Psi_{\tau \otimes \tau}^{L}=-\Psi_{\tau \otimes \tau}^{R}, \quad \Psi_{\sigma \otimes \sigma}^{L *} \Psi_{\sigma \otimes \sigma}^{R}=0 .
\end{gathered}
$$

The first case is the trivial boundary, and the second is the "fermionic" boundary where the field $\Psi_{\sigma \otimes \sigma}$ changes sign. The third is the "dual" boundary, which allows the coexistence of the order and disorder parameter fields $\sigma$ and $\mu$ (in the original Statistical Mechanics terminology, see [67, 30]) on either side: these are the two isomorphic but independent fields $\Psi_{\sigma \otimes \sigma}^{R}$ and $\Psi_{\sigma \otimes \sigma}^{L}$.

\section{$7 \quad$ Final Remarks}

\subsection{What is special about CFT in two dimensions?}

Quantum field theory in two dimensions offers a wealth of algebraic methods for the construction of models, especially conformal models. Why are these methods so efficient in two dimensions, and can one put them into perspective with QFT in four dimensions?

1. The prominent reason is the kinematical simplicity of CFT in 2D, especially the existence of chiral fields related to conserved tensors fields. Chiral fields live in a "one-dimensional spacetime", namely the light ray, which shares crucial features of space and time: local commutativity and spectral positivity of the generator of translations.

As a consequence, chiral commutators are ultralocal ( $\delta$ functions), supported only in coinciding points rather than in or on a lightcone). Based on this feature, the Lüscher-Mack theorem provides an explicit form of the possible commutators of the stress-energy tensor, with the central charge as the only free 
parameter. A similar parametrization of the commutator in higher dimensions, or without conformal symmetry, is not known.

Moreover, the algebra of the stress-energy tensor field (and also of chiral fields of scaling dimension 1) is that of an infinite-dimensional Lie algebra, permitting the application of highest-weight representation methods for the efficient study of its positive-energy representation theory. In contrast, Lie fields in 4D (whose commutators are linear in the field) don't exist [3]. (The argument, based on geometric properties of lightcones and the spectrum condition, was worked out only for scalar fields, but is presumably true more generally.)

2. Another consequence of the ultrolocal commutation relations of chiral free Fermi fields is the feature that chiral gauge transformations $\psi(x) \mapsto e^{i \alpha(x)} \psi(x)$ are automorphisms of the free Fermi (CAR) algebra. Hence, current fields are their infinitesimal generators, and can be constructed algebraically by exploiting this property.

In contrast, in $D \geq 2$, the free Fermi algebra is not gauge invariant, and gauge invariance requires the coupling to a gauge field. Currents are generators of gauge transformations at a fixed time only. Notice, however, that in two dimensions the massless free Dirac field $\psi$ is nothing but a pair of two chiral Fermi fields $\psi_{ \pm}(t \pm x)=P_{ \pm} \psi(t, x)$, where $P_{ \pm}=\frac{1}{2}\left(1 \pm \gamma_{0} \gamma_{1}\right)$. While local gauge transformations of the general form $\psi(t, x) \mapsto e^{i\left(\alpha(t, x)+\beta(t, x) \gamma_{0} \gamma_{1}\right.} \psi(t, x)$ do not preserve the equation of motion, the chiral gauge transformations are of the more special form $\psi(t, x) \mapsto e^{i\left(\alpha_{+}\left(x^{+}\right) P^{+}+\alpha_{-}\left(x^{-}\right) P^{-}\right.} \psi(t, x)$. The latter commute with $\gamma^{0} \gamma^{\mu} \partial_{\mu}=P_{+} \partial_{-}+P_{-} \partial_{+}$, hence preserve the free equation of motion and the commutation relations at all times. In fact, they may be regarded as gauge transformations of the Cauchy data. Thus, the chiral gauge transformations are the subgroup of the gauge group which is a symmetry without the interaction with a gauge field.

3. For general conformal fields in two dimensions, the chiral factorization manifests itself (in rational theories) in the form of the conformal block decomposition of their correlation functions 4. The analytic behaviour of conformal blocks under field exchange can be formulated algebraically as an "exchange algebra" of chiral components, which are in turn most naturally understood in terms of charged intertwiners among the chiral sectors subject to braid group statistics [27, II].

It is worth a remark that also in four dimensions, conformal partial waves - which are akin to but not exactly the same as conformal blocks - exhibit a factorization into "chiral" factors [24]. This feature has been exploited for the study of Wightman positivity (positivity of the Hilbert space inner product defined by $2 n$-point correlation functions) [60, 59]; but the algebraic counterpart of an underlying exchange algebra, as suggested in [66], has not been identified. 
4. Many classification results obtained in two-dimensional conformal QFT have been obtained thanks to rationality (finitely many positive-energy representations), or related properties (strong additivity, split property, finite $\mu$ index) in the AQFT framework. These results are then owing to the ensuing rigidity of the DHR category with finitely many irreducible sectors.

QFT models with finitely many sectors exist also in four dimensions - e.g., whenever the global gauge group as in Sect.6.1 is a finite group. The corresponding classification results, also in the case of compact gauge groups, are all in terms of groups and subgroups, and do not exhibit a comparably rich structure as in the low-dimensional case with sectors with braid group statistics.

5. Recall that every local QFT can be encoded in a Borchers triple $(M, \Omega, U)$, see Sect. 6.3. The defining properties of Borchers triples include the proper adjoint action of the representation $U$ of the Poincaré group on the von Neumann algebra $M$. In four dimensions, these are quite difficult to satisfy, and compelling ideas how to construct such triples (if one does not want to start from a QFT) are lacking. In contrast, in two dimensions, only a posive-energy representation of the translation group is required, and the representation of the Poincare group can be constructed from the former with Modular Theory (Borchers' Theorem), and its required properties are automatic. Therefore, Borchers triples are much easier to obtain in two dimensions, and their data can be subjected to algebraic deformations while preserving their defining properties. This appears to be a promising non-perturbative approach to obtain new QFT models by deformation of given ones. This idea does not require conformal symmetry.

\subsection{What can we learn for QFT in four dimensions?}

Algebraic QFT is a powerful approach that conceptually clarifies many features known to be true in QFT models, or expected to be true in QFT in general. Its value is not least that it allows to sharply exhibit the (in other approaches often tacit) assumptions that are responsible for these features. At the same time, it allows to investigate the consequences when some of these assumptions are not fulfilled - be it systematically due to the structure of the two-dimensional spacetime and its symmetry group, or model-dependently due to specific properties of the dynamics.

The "bifurcation" between four dimensions and two dimensions has particularly strong consequences in the theory of superselection sectors, where the braided tensor category is completely degenerate in four dimensions, and maximally non-degenerate (modular) in large classes of two-dimensional conformal QFT models. Yet, it is rewarding to view either extreme in the light of the 
other, or in the context of the general structure, as this opens the mind to the many options QFT has in store that might be missed by model studies.

Once the underlying abstract structure has been identified (and separated from the dynamical details of specific models), the road is open to classifications. Many classifications have been obtained, mostly of representation theoretic nature.

Let us return to the kinematic simplicity of QFT in two dimensions, with the Poincaré group being a subgroup of the product of two translation-dilation groups. Representations of the translation-dilation group with positive generator of the translations can be constructed by Modular Theory 69. This feature is exploited in new algebraic deformation approaches. The passage to four dimensions is presently not yet very satisfactory; e.g., the "warped convolutions" deformations [17] break parts of the Lorentz symmetry. In contrast, the modular approach indicated in [38] seems not very practical, but it points out a direction: The problem is to control the relations between the many translationdilation subgroups that generate the Poincaré group. This is reminiscent of the classification of semisimple Lie algebras by controlling the relations between the many $s u(2)$ subalgebras that generate them. Gaining experience with twodimensional models, one may expect progress also in four dimensions.

Acknowledgment. I thank Yoh Tanimoto and Jakob Yngvason for a critical reading of the manuscript. Supported by the German Research Foundation (Deutsche Forschungsgemeinschaft (DFG)) through the Institutional Strategy of the University of Göttingen. The hospitality and support of the Erwin Schrödinger International Institute for Mathematical Physics, Vienna, is gratefully acknowledged.

\section{References}

[1] S. Alazzawi, Deformation of fermionic quantum field theories and integrable models, Lett. Math. Phys. 103, 37-58 (2012).

[2] S. Alazzawi, Deformation of quantum field theories and the construction of interacting models, Ph.D. thesis, University of Vienna (2015).

[3] K. Baumann, There are no scalar Lie fields in three or more dimensional space-time, Commun. Math. Phys. 47, 69-74 (1976).

[4] A.A. Belavin, A.M. Polyakov, A.B. Zamolodchikov, Infinite conformal symmetry in two-dimensional quantum field theory, Nucl. Phys. B 241, 333-380 (1984). 
[5] M. Bischoff, Y. Kawahigashi, R. Longo, Characterization of $2 D$ rational local conformal nets and its boundary conditions: the maximal case, arXiv:1410.8848.

[6] M. Bischoff, Y. Kawahigashi, R. Longo, K.-H. Rehren: Phase boundaries in algebraic conformal QFT, arXiv:1405.7863.

[7] M. Bischoff, Y. Kawahigashi, R. Longo, K.-H. Rehren: Tensor categories and endomorphisms of von Neumann algebras. With applications to quantum field theory, SpringerBriefs in Mathematical Physics, Vol. 3, 2015, arXiv:1407.4793v3.

[8] M. Bischoff, Y. Tanimoto, Construction of wedge-local nets of observables through Longo-Witten endomorphisms. II, Commun. Math. Phys. 317, 667695 (2013).

[9] J. Böckenhauer, D. Evans, Modular invariants, graphs and $\alpha$-induction for nets of subfactors. II, Commun. Math. Phys. 200, 57-103 (1999).

[10] J. Böckenhauer, D. Evans, Y. Kawahigashi, On $\alpha$-induction, chiral projectors and modular invariants for subfactors, Commun. Math. Phys. 208, 429-487 (1999).

[11] J. Böckenhauer, D. Evans, Y. Kawahigashi, Chiral structure of modular invariants for subfactors, Commun. Math. Phys. 210, 733-784 (2000).

[12] H.-J. Borchers, The CPT-theorem in two-dimensional theories of local observables, Commun. Math. Phys. 143, 315-332 (1992).

[13] R. Brunetti, D. Guido, R. Longo, Modular structure and duality in conformal quantum field theory, Commun. Math. Phys. 156, 201-219 (1993).

[14] R. Brunetti, D. Guido, R. Longo, Modular localization and Wigner particles, Rev. Math. Phys. 14, 759-785 (2002).

[15] D. Buchholz, K. Fredenhagen, Locality and the structure of particle states, Commun. Math. Phys. 84, 1-54 (1982).

[16] D. Buchholz, R. Haag, The quest for understanding in relativistic quantum physics, J. Math. Phys. 41, 3674-3697 (2000).

[17] D. Buchholz, G. Lechner, S.J. Summers, Warped convolutions, Rieffel deformations and the construction of quantum field theories, Commun. Math. Phys. 304, 95-123 (2011).

[18] D. Buchholz, G. Mack, I. Todorov, The current algebra on the circle as a germ of local field theories, Nucl. Phys. B (Proc. Suppl.) 5B, 20-56 (1988).

[19] D. Buchholz, J.E. Roberts, New light on infrared problems: Sectors, statistics, symmetries and spectrum, Commun. Math. Phys. 330, 935-972 (2014).

[20] D. Buchholz, E. Wichmann, Causal independence and energy-level density in quantum field theory, Commun. Math. Phys. 106, 321-344 (1986).

[21] A. Cappelli, C. Itzykson, J.-B. Zuber, The A-D-E classification of minimal and $A_{1}^{(1)}$ conformal invariant theories, Commun. Math. Phys. 113, 1-26 (1987). 
[22] S. Carpi, Y. Kawahigashi, R. Longo, How to add a boundary condition, Commun. Math. Phys. 322, 149-166 (2013).

[23] S. Carpi, Y. Kawahigashi, R. Longo, M. Weiner, From vertex operator algebras to conformal nets and back, arXiv:1503.01260.

[24] F.A. Dolan, H. Osborn, Conformal four point functions and the operator product expansion, Nucl. Phys. B 599, 459-496 (2001).

[25] S. Doplicher, R. Haag, J.E. Roberts, Local observables and particle statistics. I, Commun. Math. Phys. 23, 199-230 (1971), and II, Commun. Math. Phys. 35, 49-85 (1974).

[26] S. Doplicher, J.E. Roberts, Why there is a field algebra with a compact gauge group describing the superselection structure in particle physics, Commun. Math. Phys. 131, 51-207 (1990).

[27] K. Fredenhagen, K.-H. Rehren, B. Schroer, Superselection sectors with braid group statistics and exchange algebras. I, Commun. Math. Phys. 125, 201-226 (1989), and II, Rev. Math. Phys. SI1 (Special Issue) 113157 (1992).

[28] J.B. Frenkel, V.G. Kac, Basic representations of affine Lie algebras and dual resonance models, Invent. Math. 62, 23-66 (1980).

[29] D. Friedan, Z. Qiu, and S. Shenker, Conformal invariance, unitarity, and critical exponents in two dimensions Phys. Rev. Lett. 52, 1575-1578 (1984).

[30] J. Fröhlich, J. Fuchs, I. Runkel, C. Schweigert, Kramers-Wannier duality from conformal defects, Phys. Rev. Lett. 93, 070601 (2004).

[31] J. Fröhlich, J. Fuchs, I. Runkel, C. Schweigert, Correspondences of ribbon categories, Ann. Math. 199 192-329, (2006).

[32] P. Goddard, A. Kent, D. Olive, Unitary representations of the Virasoro and super-Virasoro algebras, Commun. Math. Phys. 103, 105-119 (1986).

[33] D. Guido, R. Longo, The conformal spin and statistics theorem, Commun. Math. Phys. 181, 11-35 (1996).

[34] D. Guido, R. Longo, H.-W. Wiesbrock, Extensions of conformal nets and superselection structures, Commun. Math. Phys. 192, 217-244 (1998).

[35] R. Haag: Local Quantum Physics, Springer Verlag, Berlin - Heidelberg New York, 1996.

[36] M. Izumi, H. Kosaki, On a subfactor analogue of the second cohomology, Rev. Math. Phys. 14, 733-737 (2002).

[37] V.G. Kac: Infinite dimensional Lie algebras, Cambridge University Press, 1985.

[38] R. Kähler, H.-W. Wiesbrock, Modular theory and the reconstruction of four-dimensional quantum field theories, J. Math. Phys. 42, 74-86 (2001).

[39] Y. Kawahigashi, R. Longo, Classification of local conformal nets. Case $c<1$, Ann. Math. 160, 493-522 (2004). 
[40] Y. Kawahigashi, R. Longo, Classification of two-dimensional local conformal nets with $c<1$ and 2-cohomology vanishing for tensor categories, Commun. Math. Phys. 244, 63-97 (2004).

[41] Y. Kawahigashi, R. Longo, Local conformal nets arising from framed vertex operator algebras, Adv. Math. 206, 729-751 (2006).

[42] Y. Kawahigashi, R. Longo, M. Müger, Multi-interval subfactors and modularity of representations in conformal field theory, Commun. Math. Phys. 219, 631-669 (2001).

[43] A. Kirillov Jr., V. Ostrik, On q-analog of McKay correspondence and ADE classification of sl(2) conformal field theories, Adv. Math. 171, 183-227 (2002).

[44] Ch. Köhler, On the localization properties of quantum fields with zero mass and infinite spin, Ph.D. thesis, University of Vienna (2015).

[45] L. Kong, I. Runkel, Morita classes of algebras in modular tensor categories, Adv. Math. 219, 1548-1576 (2008).

[46] G. Lechner, Construction of quantum field theories with factorizing Smatrices, Commun. Math. Phys. 277, 821-860 (2008).

[47] G. Lechner, Algebraic constructive quantum field theory: Integrable models and deformation techniques, arXiv:1503.03822.

[48] G. Lechner, J. Schlemmer, Y. Tanimoto, On the equivalence of two deformation schemes in quantum field theory, Lett. Math. Phys. 103, 421-437 (2013).

[49] R. Longo, Conformal subnets and intermediate subfactors, Commun. Math. Phys. 237, 7-30 (2003).

[50] R. Longo, V. Morinelli, K.-H. Rehren, Where infinite-spin particles are localizable, arXiv:1505.01759.

[51] R. Longo, K.-H. Rehren, Nets of subfactors, Rev. Math. Phys. 7, 567-597 (1995).

[52] R. Longo, K.-H. Rehren, Local fields in boundary CFT, Rev. Math. Phys. 16, 909-960 (2004).

[53] R. Longo, K.-H. Rehren, How to remove the boundary in CFT - an operator algebraic procedure, Commun. Math. Phys. 285, 1165-1182 (2009).

[54] R. Longo, E. Witten, An algebraic construction of boundary quantum field theory, Commun. Math. Phys. 303, 213-232 (2011).

[55] R. Longo, F. Xu, Topological sectors and a dichotomy in conformal field theory, Commun. Math. Phys. 251, 321-364 (2004).

[56] M. Lüscher, G. Mack, Global conformal invariance in Quantum Field Theory, Commun. Math. Phys. 41, 203-234 (1975).

[57] G. Mack, Introduction to conformal invariant quantum field theory in two and more dimensions, in: Nonperturbative QFT, eds. G. t Hooft et al., pp. 353-383 (Plenum Press, 1988). 
[58] J. Mund, B. Schroer, J. Yngvason, String-localized quantum fields and modular localization, Commun. Math. Phys. 268, 621-672 (2006).

[59] C. Neumann, K.-H. Rehren, L. Wallenhorst, New methods in conformal partial wave analysis, V. Dobrev (ed.), Lie Theory and Its Applications in Physics: IX International Workshop, Springer Proceedings in Mathematics and Statistics 36, pp. 109-126 (2013).

[60] N.M. Nikolov, K.-H. Rehren, I.T. Todorov, Partial wave expansion and Wightman positivity in conformal field theory, Nucl. Phys. B 722 [PM], 266-296 (2005).

[61] A. Pressley, G. Segal, Loop Groups, Oxford University Press, Oxford, 1986.

[62] K.-H. Rehren, Weak $C^{*}$ Hopf symmetry, in: Quantum Groups Symposium at "Group21", eds. H.-D. Doebner et al., Goslar 1996 Proceedings, Heron Press, Sofia (1997), pp. 62-69, arXiv:q-alg/9611007.

[63] K.-H. Rehren, Canonical tensor product subfactors, Commun. Math. Phys. 211, 395-406 (2000).

[64] K.-H. Rehren, Algebraic holography, Ann. H. Poinc. 1, 607-623 (2000).

[65] A. Schellekens, N. Warner: Conformal subalgebras of Kac-Moody algebras, Phys. Rev. D 34, 3092-3096 (1986).

[66] B. Schroer, Braided structure in 4-dimensional conformal quantum field theory, Phys. Lett. B 506, 337-343 (2001).

[67] B. Schroer, T.T. Truong, The order/disorder quantum field operators associated with the two-dimensional Ising model in the continuum limit, Nucl. Phys. B 144, 80-122 (1978).

[68] C.-P. Staszkiewicz, Die lokale Struktur abelscher Stromalgebren auf dem Kreis, PhD Thesis (in German), Freie Universität Berlin, 1995.

[69] M. Takesaki: Theory of Operator Algebras II, Springer Encyclopedia of Mathematical Sciences, Vol. 125 (2003).

[70] Y. Tanimoto, Construction of wedge-local nets of observables through Longo-Witten endomorphisms, Commun. Math. Phys. 314, 443-469 (2012).

[71] Y. Tanimoto, Construction of two-dimensional quantum field theory models through Longo-Witten endomorphisms, Forum of Math., Sigma 2, e7 (2014), arXiv:1301.6090.

[72] E. Verlinde, Fusion rules and modular transformations in $2 D$ conformal field theory, Nucl. Phys. B 300, 360-376 (1988).

[73] A. Wassermann, Kac-Moody and Virasoro algebras, arXiv:1004.1287.

[74] F. Xu, Mirror extensions of local nets, Commun. Math. Phys. 270, 835-847 (2007). 